\title{
Interference Detection and Characterization with an Array based GNSS Receiver using Conformal Antennas in Maritime Environments
}

\author{
A. Konovaltsev ${ }^{1}$, S. Caizzone ${ }^{1}$, K. Yinusa ${ }^{1}$, M. Sgammini ${ }^{1}$, E. Pérez Marcos ${ }^{1}$, M. Appel ${ }^{1}$, \\ M. Cuntz ${ }^{1}$, W. Elmarissi ${ }^{1}$, M. Meurer ${ }^{1,2}$, \\ ${ }^{1}$ German Aerospace Center (DLR) \\ ${ }^{2}$ Chair of Navigation, RWTH Aachen University, Germany
}

\section{BIOGRAPHIES}

Andriy Konovaltsev received his engineer diploma and the Ph.D. degree in electrical engineering from Kharkov State Technical University of Radio Electronics, Ukraine in 1993 and 1996, respectively. He joined the Institute of Communications and Navigation of DLR in 2001. His main research interest is in application of antenna array signal processing for improving performance of satellite navigation systems in challenging signal environments.

Stefano Caizzone received the M.Sc. in Telecommunications Engineering and the Ph.D. degree in Geoinformation from the University of Rome "Tor Vergata", Italy, in 2009 and 2015, respectively. Since 2010, he is with the Antenna group of the Institute of Communications and Navigation of the German Aerospace Center (DLR), Wessling, Germany, where he is responsible for the development of innovative miniaturized antennas. His main research interests concern small antennas for RFIDs and satellite navigation, antenna arrays and grids with enhanced sensing capabilities, controlled radiation pattern antennas for robust satellite navigation and high-performance antenna design for precise satellite navigation. He is a participant of RTCA SC-159, active in the definition of minimum operational performance specifications for avionic GNSS equipment.

Kazeem Yinusa obtained his BSc in electronics and communications engineering from the Arab Academy for Science and Technology in Cairo in 2009. From 2009 till 2015, he studied at the Technical University Munich where he obtained his MSc in communications engineering and his $\mathrm{PhD}$ in electrical engineering based on his dissertation on echo suppression techniques for near-field antenna measurements. He joined the Institute of Communications and Navigation at DLR in March 2015 as a researcher with main interest in antenna technologies for satellite navigation.

Matteo Sgammini received the BEng degree in electrical engineering in 2005 from the University of Perugia. He joined the Institute of Communications and Navigation of the German Aerospace Center (DLR) in 2008. His field of research is interference detection and mitigation for global navigation satellite systems.

Emilio Pérez Marcos studied Electrical Engineering at Valladolid University, Spain. From 2006 to 2008 he was granted a young research position within the Electronics Department at the same university. From 2009 to 2014 he worked as Research Engineer in the Medical Technology Industry in Jena, Germany. Since 2014 he has been working within the Algorithms and End-Devices group in the Institute of Communications and Navigation at DLR Oberpfaffenhofen. His current research topics include signal disturbances, interferences and cybersecurity in real time GNSS receivers and Spatial-Temporal Adaptive Processing techniques for multi antenna systems.

Manuel Appel received his diploma degree in electrical engineering from the university of applied science Ingolstadt, Germany in 2008. Additionally he received a M.Sc. degree from Technical University Munich in 2013 after working at Fraunhofer Institute for Integrated Circuits in Erlangen. He joined the Institute for Communication and Navigation of DLR in January 2014. His main research interest is in development of signal processing algorithms for robust GNSS receivers with the main focus on spoofing detection and mitigation.

Manuel Cuntz received the diploma in electrical engineering degree in 2005 from the Technical University of Kaiserslautern. He joined the Institute of Communications and Navigation of DLR in June 2006. His fields of research are multi-antenna satellite navigation receivers. 
Wahid Elmarissi received the B.Sc. in Physics from the University of Sciences "Mohammed V", Rabat, Morocco, in 1992 and the Dipl. Ing. (FH) in electrical engineering from the University of Applied Sciences, Kiel, Germany, in 2003. In 2003, he joined the Institute of Communications and Navigation of the German Aerospace Center (DLR), where is responsible for measurement and manufacturing of antennas and antenna electronics.

Michael Meurer received the diploma in electrical engineering and the Ph.D. degree from the University of Kaiserslautern, Germany. After graduation, he joined the Research Group for Radio Communications at the Technical University of Kaiserslautern, Germany, as a senior key researcher, where he was involved in various international and national projects in the field of communications and navigation both as project coordinator and as technical contributor. From 2003 till 2013, Dr. Meurer was active as a senior lecturer and Associate Professor (PD) at the same university. Since 2006 Dr. Meurer is with the German Aerospace Centre (DLR), Institute of Communications and Navigation, where he is the director of the Department of Navigation and of the center of excellence for satellite navigation. In addition, since 2013 he is a professor of electrical engineering and director of the Chair of Navigation at the RWTH Aachen University. His current research interests include GNSS signals, GNSS receivers, interference and spoofing mitigation and navigation for safety-critical applications.

\begin{abstract}
The results of the interference measurement campaign carried out on board of a large container ship over the timespan of 2.5 months are presented. The interference situation in L1/E1 and L5/E5a frequency bands of GPS/Galileo that is representative for many maritime signal environments is characterized by observing the variation of the received IF power. Four detected interference events in L1/E1 band are presented. The interference effect of the signals of Distance Measurement Equipment (DME) in L5/E5a band is assessed. The characteristics of observed man-made radio emissions in both frequency bands are analyzed by using the high-rate recorded data. The design of the measurement system and its components is discussed in details.
\end{abstract}

\title{
INTRODUCTION
}

The navigation signals of global navigation satellite systems (GNSS), such as GPS and Galileo, are received by the user antenna with a power as small as $-154 \mathrm{dBW}$ or less. This is the reason for the inherent susceptibility of the GNSS services to radio frequency interference (RFI). The distortion and operational outages of the navigation receivers caused by this phenomenon pose a serious threat for many applications of GNSS, especially for the ones with safety-critical aspects or big economical impact. High vulnerability of the maritime navigation systems to deliberate GNSS interference, specifically jamming, was demonstrated by several studies in the past [1][2]. The development of adequate countermeasures is one of the topics addressed by the e-Navigation strategy launched by the International Maritime Organization (IMO)[3]. One of the proposed solutions to reduce the vulnerability of the next generation of GNSS receivers is the utilization of additional signals and frequency bands [4].

The development of adequate countermeasures against radio frequency interference, deliberate or unintentional, requires the knowledge about the typical signal environments encountered by a shipborne GNSS receiver. However, the most of the information available about the observed RFI signals and assessed interference effect is representative for the GNSS receivers used in aviation [5] or in the land-based applications. Only little information is currently available about the signal situation in maritime environments and mostly regarding the RFI events occurred in harbors [5][6]. In order to close this gap the Institute of Communications and Navigation of the German Aerospace Center (DLR) carries out a worldwide measurement campaign by installing the data collection equipment on board of a large container ship. This campaign is a part of the nationally funded project siNafa. A multi-antenna interference detection and recording system was developed by DLR which is capable of detecting the presence of interference signals and automatically recording data snapshots in case of such interfering events. The paper describes the design of the developed measurement system and provides details about its individual components. Further, the results for the interference situation in L1/E1 and L5/E5a band are presented. These results have been obtained by analyzing the data collected during the first run of the measurements which took place between April, 20 and July, 12 of 2017.

\section{MEASUREMENT SYSTEM}

A simplified block diagram of the measurement system is presented in Figure 1. The key elements of the system are a 7element conformal antenna array receiving signals in GPS/Galileo L1/E1 and L5/E5a frequency bands, two RF front-ends, one for each frequency band, and a recorder of signal snapshots that is triggered by an interference detection algorithm. Because of large amount of recorded data, the signal snapshots are saved on an external RAID storage with the sufficient capacity. 
Additionally, a multi-antenna GNSS receiver is used in parallel to the snapshot recorder in order to collect reference data for the analysis of the actual interference effect. In order to avoid any need for the maintenance of such an experimental system from the side of the crew of the container ship, corresponding measures has to be taken. One of these measures is the use of an uninterruptible power supply unit for overcoming short outages in the onboard power network. Also, an additional data link over satellite communication is used for the periodical remote checks of the operation status and eventual re-configuration of the system components. The installation of the system components on the board of the container ship (see Figure 2) of the Post-Panamax class operated by the Hapag Lloyd Company, an associated partner of the siNafa project, is shown in Figure 3. The antennas (see Figure 2a) were placed at the deck above the navigation deck. The 19-inch rack shown in Figure $2 \mathrm{~b}$ was installed in a side room at the level of the navigation deck. In the following the individual components of the measurement systems will be presented in more details.

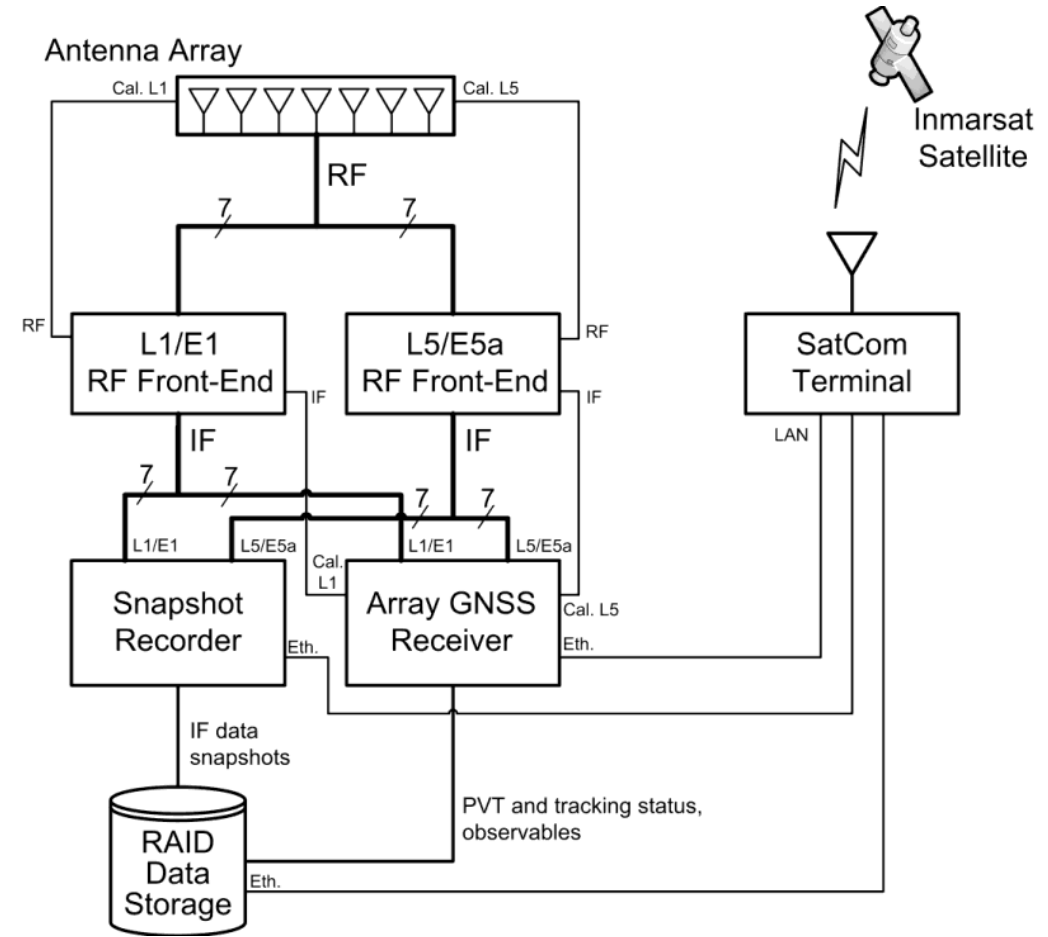

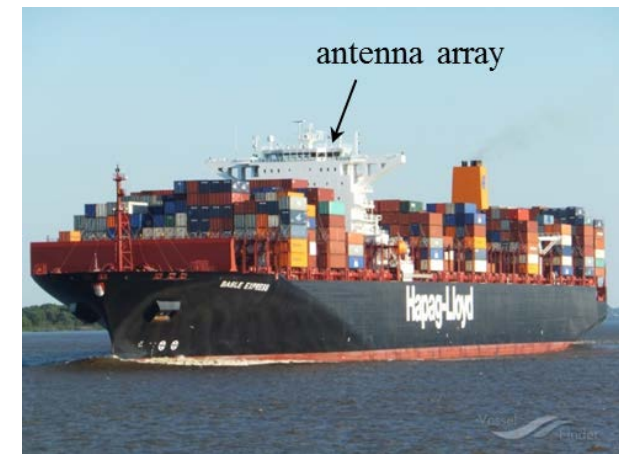

Figure 2 Container ship carrying the measurement system (photo from vesselfinder.com)

Figure 1 Block diagram of measurement system

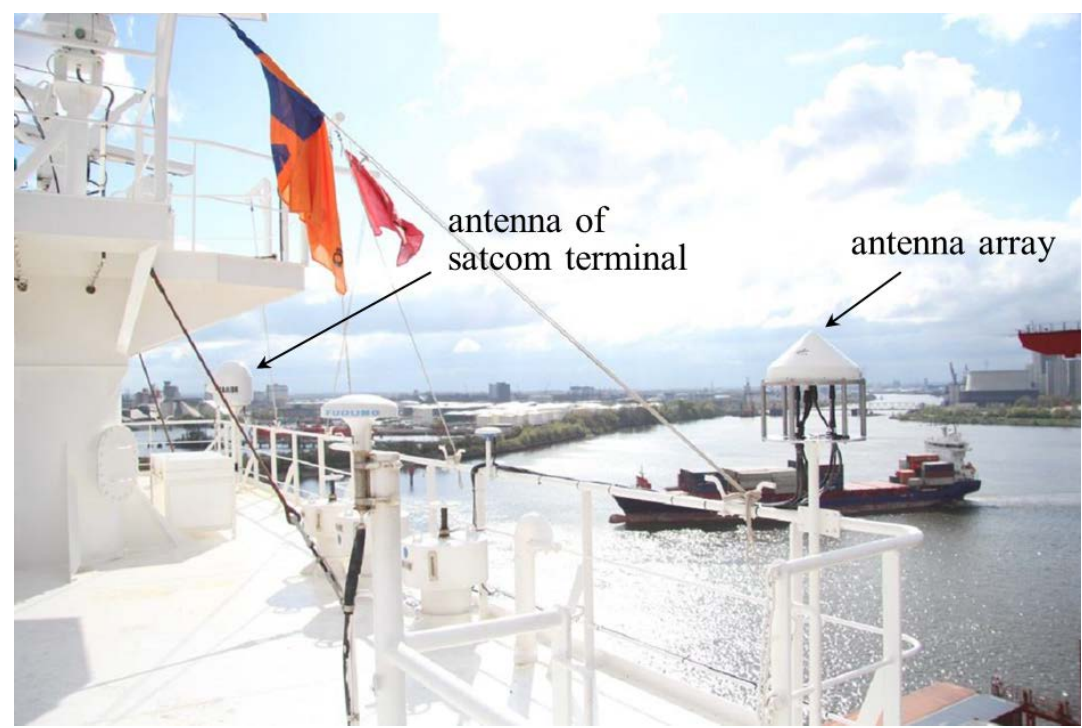

a) antenna array and SatCom antenna

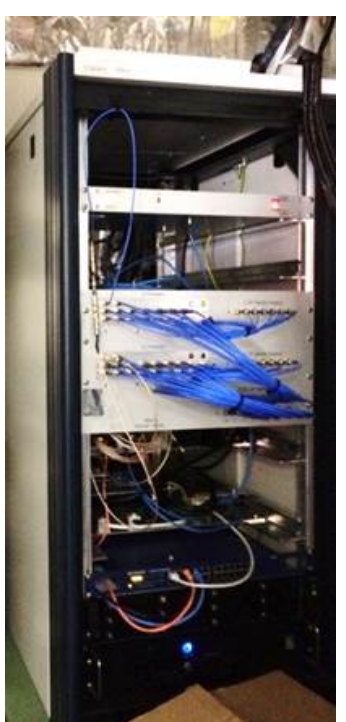

b) 19-inch rack with RF front ends, snapshot recorder, array receiver, SatCom terminal, RAID storage and UPS

Figure 3 Installation places of individual hardware components 


\section{Antenna Array}

The most distinctive feature of the proposed measurement system (see Figure 1) is the use of a conformal antenna array for signal reception. The utilization of the antenna array allows characterizing interference in spatial domain and easier distinguishing between multiple overlaying signals by using their different angles of arrival. The antenna array has a hemispheric form that enables it to achieve a low gain drop-off and good signal reception at low elevation angles. The array consists of seven elements arranged on a spherical cap with an equatorial diameter of 24 cm. Coverage of the upper hemisphere is achieved by arranging the antenna elements such that one element is placed on the top of the spherical cap and the remaining six elements are uniformly arranged on a ring below the top element and are radially directed at an angle of 58 degrees from zenith (see Figure 4). In order to enable multi-frequency and multi-constellation GNSS signal reception, the antenna elements utilize a stacked patch configuration which results in resonance at Galileo E5alb and E1 frequencies. The frequency response of a single antenna element of the array is shown in Figure 5.

In order for an antenna array to function properly in a multi-antenna navigation receiver, the antenna has to be calibrated. Calibration is usually performed for the whole signal paths of all the antenna elements including electronic components of the digital receiver. Accurate calibration of the individual signal paths compensates for the changes in the characteristics of the active components of the antenna architecture (see Figure 6) and front-ends which may arise due to temperature variations and aging. In the present of the measurement system, calibration of the array is achieved by a calibration network integrated into the antenna structure similar to the one proposed in [4]. The calibration network is based on 1:2 power dividers with $\lambda / 4$ impedance transformers in order to deliver signals with equal amplitude and phases at its outputs. In this way, the calibration network distributes the calibration signal to all antenna outputs from where the signal travels the whole signal paths behind the antenna elements.

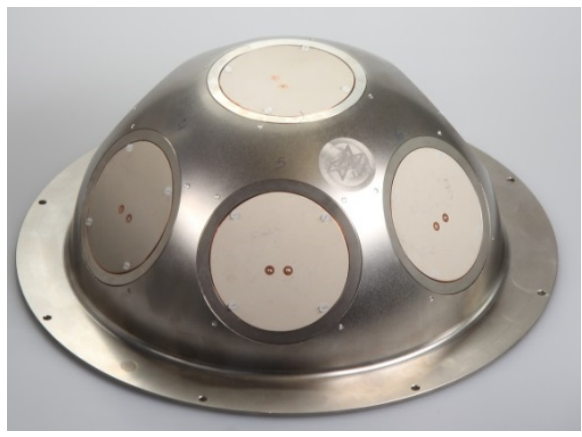

a) side view

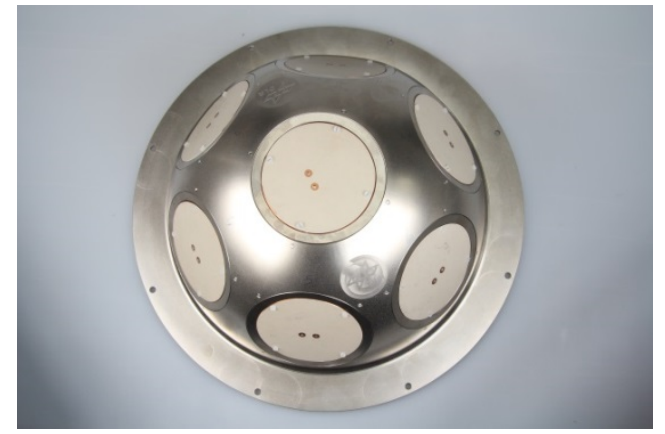

b) top view

Figure 4 Conformal antenna array

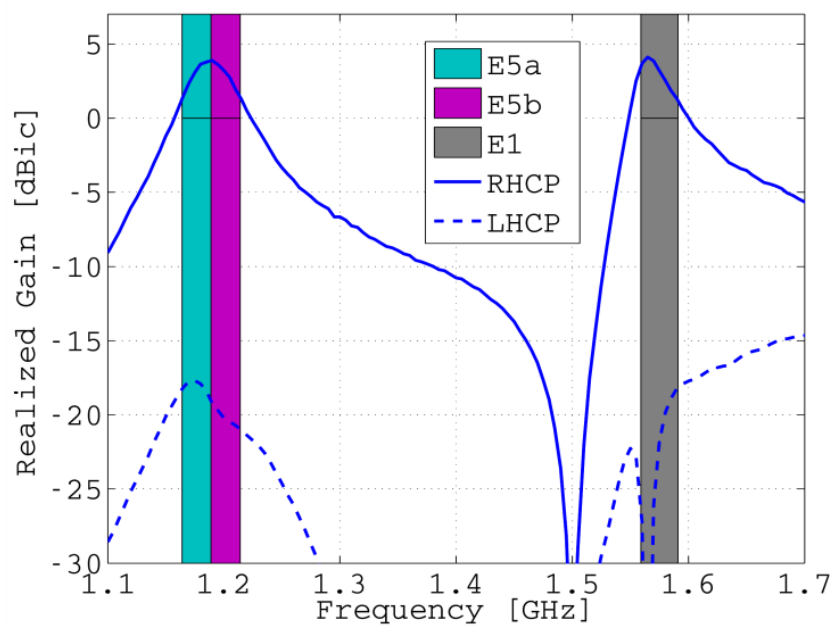

Figure 5 Frequency response of the single array element for signal arriving from zenith

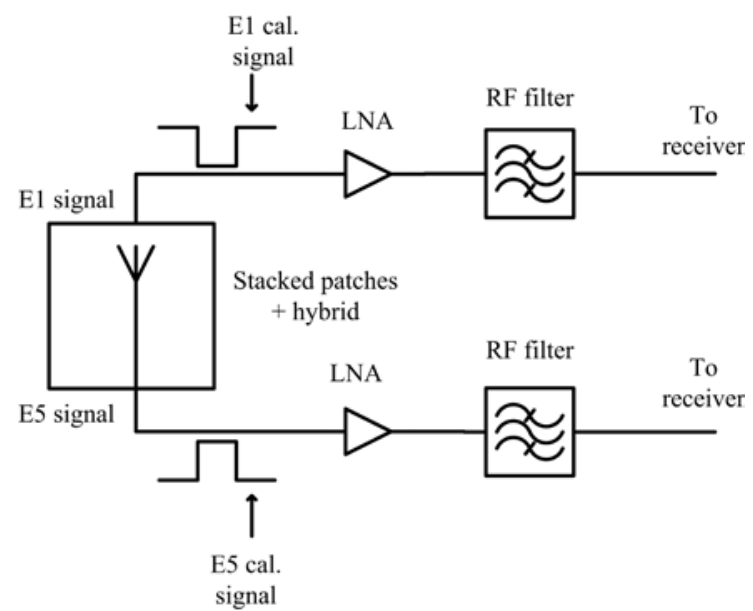

Figure 6 Signal paths for L1/E1 and L5/E5 signals after a single antenna element of the array

The simulated gain reception patterns of the array elements are shown in Figure 7 for the E1 and E5 center frequencies. It can be observed that the array can provide a relatively good coverage of the whole upper hemisphere with the laterally directed 
elements enabling the coverage of the low elevation angles. Further, in order to assess the interference effect on a receiver with single antenna, we will use the signal of the top element (element 7) of the antenna array which reception pattern is similar to the one of a common GNSS antenna.
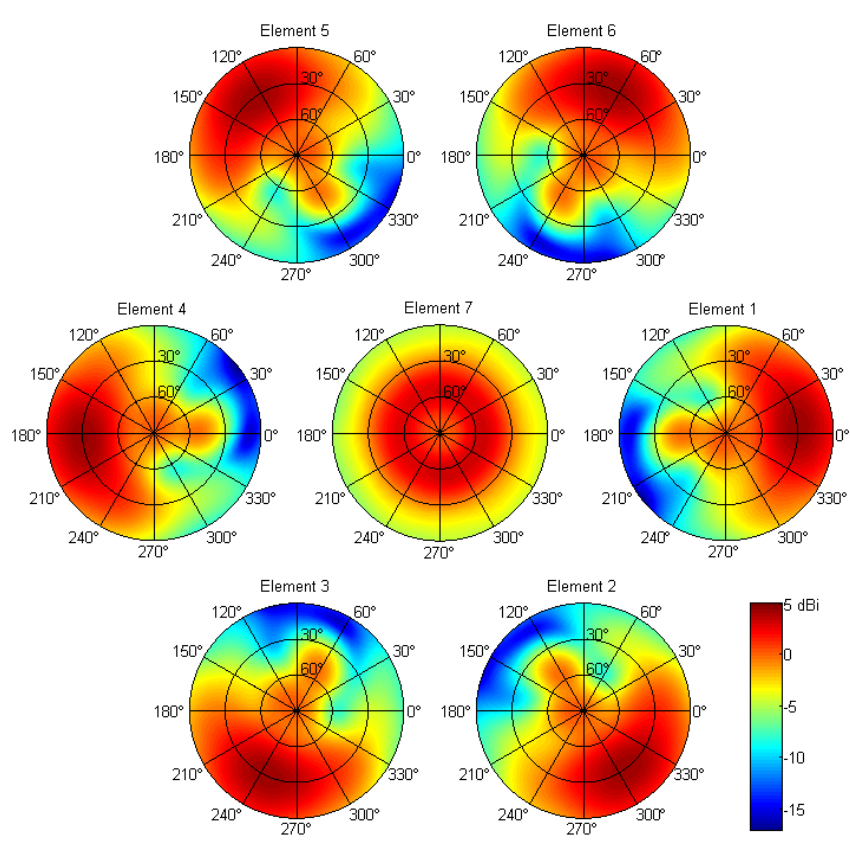

a) L1/E1 frequency
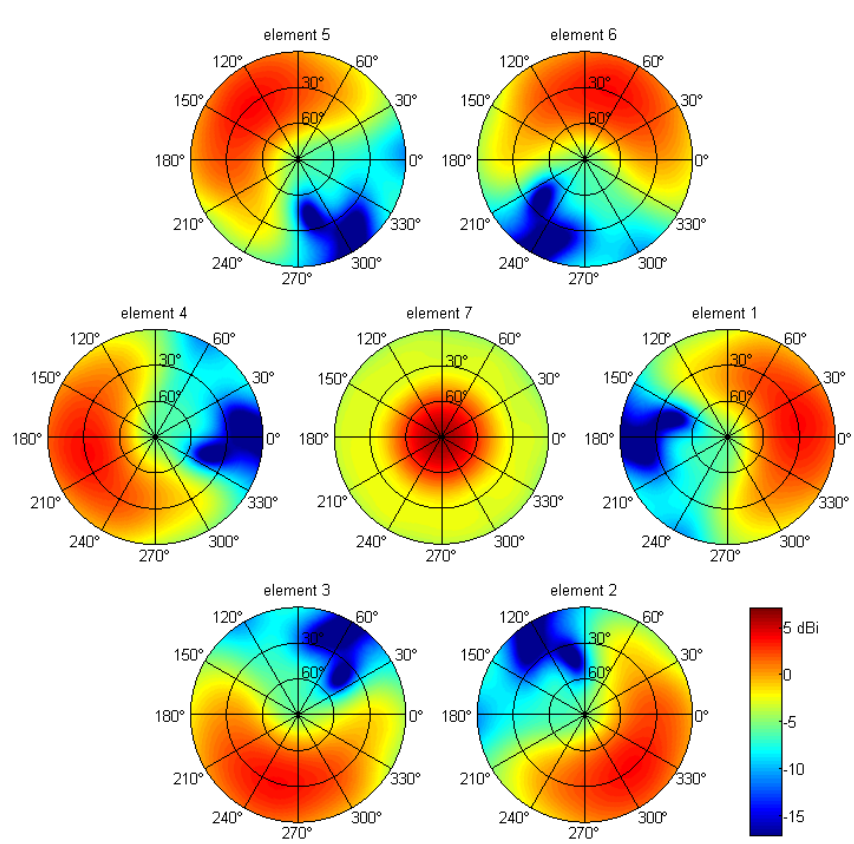

b) L5/E5a frequency

Figure 7 Simulated gain reception patterns of array elements

The E1 and E5 outputs of an antenna array element are combined together by using a power combiner so that they can be transmitted over a single RF cable to the RF front ends. The antenna array is installed at the height of about 45 meters above the sea (see Figure 3). In total eight RF cables, each of approximately 20 meter length, are used to connect the antennas with the RF front ends. Seven of these cables are used to transmit the combined E1+E5 RF outputs of the array elements. The eighth cable is used to transmit a combined E1+E5 RF calibration signal from the RF front ends to the calibration input of antenna array.

\section{L1 and L5 Front-Ends}

The seven RF signals received from the antenna array are first passed through power splitters in order to obtain the L1 and L5 signal branches (see Figure 1). The signals in the front end of each signal branch are first filtered around the corresponding carrier frequency, either L1 or L5. Further after amplification, the RF signals are down-converted to the intermediate frequency (IF) of $75 \mathrm{MHz}$ that is common for both L1 and L5 signal branches. The signal bandwidth at the output of the front end which is obtained due to the cumulative filtering effect in the entire analog signal path is approximately $20 \mathrm{MHz}$ at $\mathrm{L} 1$ branch and $24 \mathrm{MHz}$ at L5 branch. The RF front end hardware can be seen in Figure 8. The same RF front ends are also used with the DLR's GALANT demonstrator of a resilient array GNSS receiver. More details about the design of the RF front ends can be found in [3].

\section{Signal Snapshot Recorder}

The IF-outputs of the RF front-ends are split into two branches by using IF power splitters and processed in parallel by the snapshot recorder and the array L1 GPS receiver (see Figure 1). Both the snapshot recorder and the array receiver are realized by using the same hardware platform of Pico Digitizer 125 from Nutaq (see Figure 8). The platform features 16 ADCs which allows to sample all 7 array signals at both L1 and L5 frequency bands. The snapshot recorder implementation is based on the multichannel Record \& Playback IP Core for the FPGA part of the platform provided by the manufacturer. The control c++ software running on the CPU of the platform is designed to detect the presence of radio frequency interference and trigger recording of a snapshot of real-valued IF samples with 14 bit resolution of the array signals for the detailed analysis in postprocessing. The sampling rate of the IF samples is $100 \mathrm{Msps}$ which results in the digital IF of $25 \mathrm{MHz}$. The power level of the RF front end outputs is adjusted in such a way that only the amplitude range of only 3-4 bits of the ADCs is used under interference-free conditions, while the full amplitude range of the ADCs is utilized to resolve strong interference signals without suffering from the clipping effect. The length of a single data snapshot is set to 50 ms of the recording time, which results in the size of a single snapshot of $160 \mathrm{MB}$. This is also due to the fact that each signal sample is stored as a 2-byte 
integer and that the outputs of all 16 ADCS are packed in a single data block. The recorded signal data are then transferred to the RAID array (see Figure 1) with the storage capacity of 4 TB.

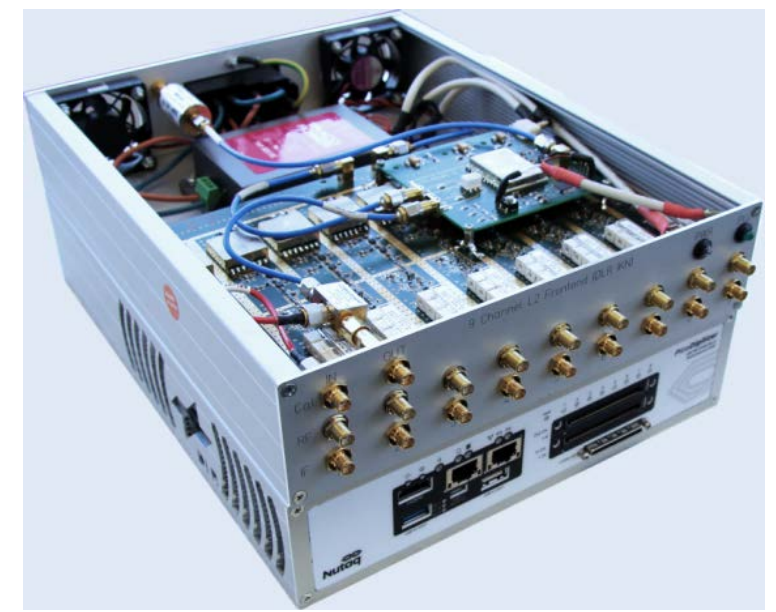

Figure 8 RF front end (top) and software-defined signal processing platform Pico Digitizer 125 of Nutaq (bottom)

The interference detection in the snapshot recorder is based on the test of the amplitudes of the IF signal samples. Because of very low power of GNSS signals it is expected that under interference-free conditions the outputs of the RF front ends are dominated by the thermal noise contributions in the signal chain and therefore the ADC samples follow the Gaussian distribution. A Gaussian distribution is defined by two parameters - a mean $\mu$ and a standard deviation $\sigma$ of the signal amplitude, i.e. $x[n] \sim \mathcal{N}(\mu, \sigma)$. Before the first use, the detection algorithm performs a calibration run where these parameters of the Gaussian signal model for the interference-free case are estimated. Also, a manual inspection of the recorded signal samples in time and frequency domains is additionally carried out during this procedure in order to assure the absence of evident radio frequency interference (RFI).

With the parameters of the Gaussian signal model being estimated, the detection of interference is performed by using M-of-N search algorithm that is commonly used for the acquisition of GNSS signals, see for example [4], p. 227. In the literature on radar signal processing this technique is typically referred to as binary integration [5]. The detection is based on testing every single sample in a data block of length $N$ for the presence of interference. The interference is declared present if at least $M$ samples deliver positive detection outcomes. The testing of a single signal sample can be seen as the following statistical test:

$$
\begin{array}{ll}
H_{0} \text { (no interference): } & x[n] \in\left[T h r_{-}, T h r_{+}\right] \\
H_{1} \text { (interference): } & x[n]<T h r_{-} \text {or } x[n]>T h r_{+}
\end{array}
$$

where two detection thresholds, $T h r_{-}$and $T h r_{+}$, are defined for the left and right sides of the Gaussian distribution with a nonzero mean value. These threshold can be computed by using the inverse cumulative distribution function, $\Phi^{-1}(\mu, \sigma, P)$, of the Gaussian distribution and the value of target false alarm probability $P_{f a, s i n g l e}$ :

$$
\begin{aligned}
& T h r=\Phi^{-1}\left(0, \sigma, 1-P_{f a, \text { single }} / 2\right) \\
& T h r_{-}=-T h r+\mu \\
& T h r_{+}=T h r+\mu
\end{aligned}
$$

The integration of the detection outcomes of the single sample tests over some observation window enables to significantly reduce the false alarm rate. In case of statistically independent single tests the resulting cumulative probability of false alarm can be expressed mathematically in term of the binomial equation [4]:

$$
P_{f a}=\sum_{n=M}^{N}\left(\begin{array}{l}
N \\
n
\end{array}\right) P_{f a, \text { single }}^{n}\left(1-P_{f a, \text { single }}\right)^{N-n}
$$

where $\left(\begin{array}{l}N \\ n\end{array}\right)$ denotes the binomial coefficient defined as $\left(\begin{array}{l}N \\ n\end{array}\right)=\frac{N !}{n !(N-n) !}$. However, the ADC samples produced by the current measurement system cannot be considered as uncorrelated because of significant oversampling factor. Therefore the settings for the parameters $M$ and $N$ in the M-of-N detection algorithm were tuned based on the results of laboratory tests and the 
actual performance in the field. Since the storage capacity is limited, it is mandatory to limit the amount of data saved each time an interference detected. The design decision was to introduce a "dead time" parameter. Once an interference is detected, and the corresponding data snapshot is recorded, no other data will be saved during the "dead time". The value for this "dead time” parameter was set to 30 seconds. Also, in order to have regular control data, a data save operation is periodically triggered independently of the interference detector. The regular control data is saved every 10 minutes.

The first implementation of the detection algorithm was performed in the FPGA part of the signal processing platform, which is very practical for achieving the fastest reaction times for triggering the Record \& Playback FPGA application. The parameters of the M-of-N detection algorithm in the first implementation were set to $P_{\text {fa,single }} \sim 1 \%, N=150$ and $M=30$. In order to allow for more flexibility in choosing the parameters of the detection algorithm as well as to enable easy addition of other detection techniques, the current implementation is based on the pulling of continuously recorded data snapshots from FPGA to CPU RAM and data analysis in the C++ software running under Linux operation system. The average transfer time to CPU RAM of a single snapshot of 4.5 seconds allows to work with the dead times which are sufficiently short for the application in hand. For example, for the used measurement set-up - 4 TB available to save the collected data, the length of a single data record of $160 \mathrm{MB}$ - the average time interval between two recordings should be kept at least at 4.5 minutes. The current implementation uses the following settings of the M-of-N detection algorithm: $P_{\text {fa,single }}=10 \%, N=1500$ and $M=300$. Another difference between these two implementations lies also in handling of the antenna diversity provided through the use of the antenna array. The second version of the detection algorithm declares an RFI event if only at least signals of two array elements generate corresponding test statistics, while with the first realization the interference detection at any of the array element was sufficient.

\section{Real-Time Receiver}

The second signal processing platform in the measurement system (see Figure 1) is used to run the real-time array receiver. The receiver is a modified version of the array receiver demonstrator developed by DLR [8] which was adjusted to handle 7 array elements and comply with the national export regulations of Germany. In this version of the receiver only GPS L1 C/A signals are used for positioning. The post-correlation adaptive beamforming provides some additional anti-jamming resistance however no active null-steering to mitigate jamming signals is utilized. The receiver was configured to save the status information about the PVT solution and satellite tracking every 20 seconds as well as to generate commonly used observables of a GNSS receiver - ranges, Doppler and C/N0 values - every second.

\section{MEASUREMENT RESULTS}

In this section the results obtained by processing the data collected during 84 full calendar days between April, 20th and July, 12th of 2017 will be presented. The route of the container ship performed during this time is shown in Figure 9.

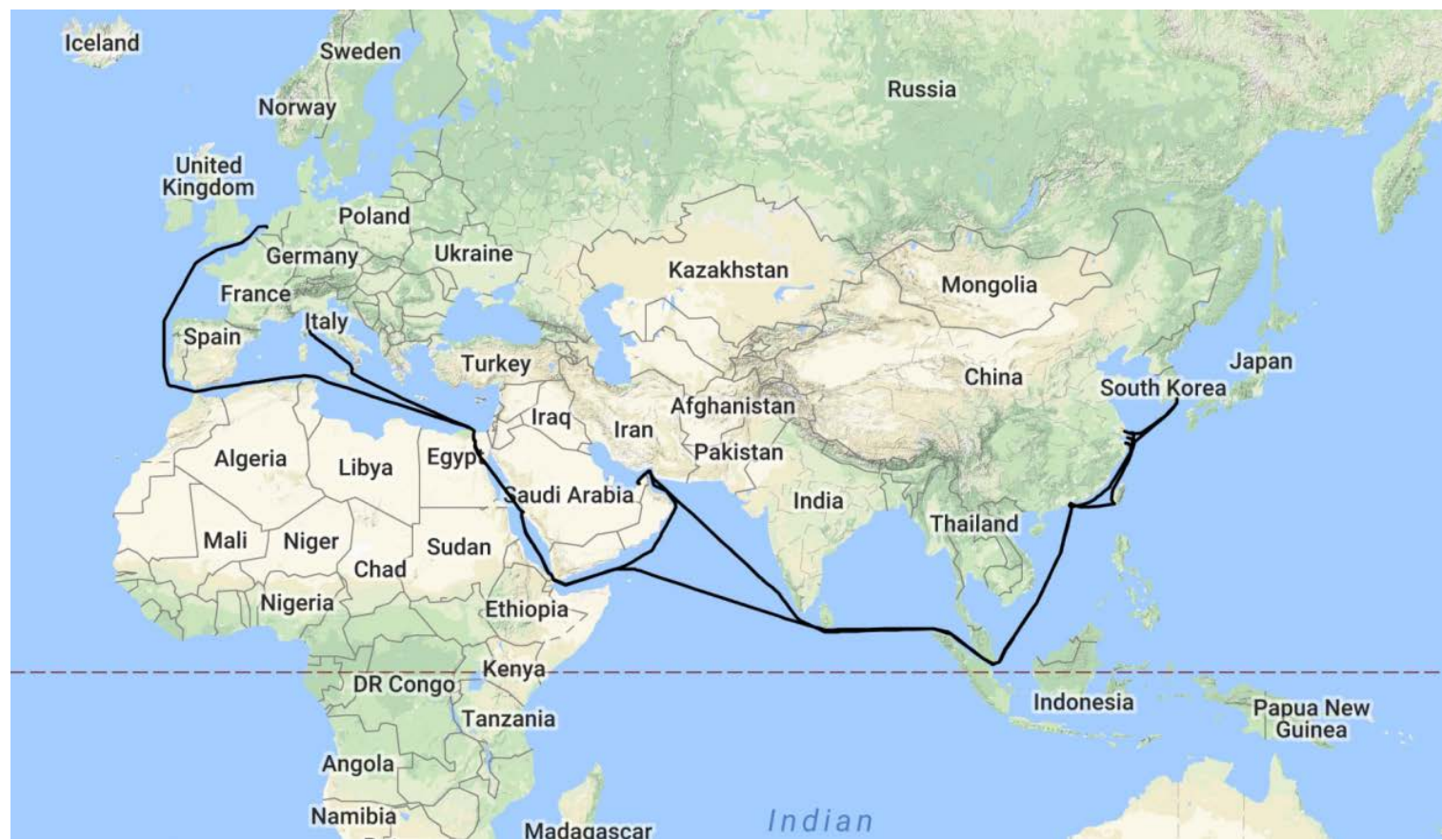

Figure 9 Route of the ship during the measurement campaign 
In total 25439 snapshots of IF samples containing the data for both L1 and L5 frequency bands have been collected. Figure 10 shows the distribution of the number of recorded snapshots over the individual days of the measurement campaign. It can be observed that the number of records per day significantly decreased after April, 26th. This is due to the adjustments of the parameters $P_{f a \text {,single }}$ and $M$ of the detection algorithm, which made it less sensitive to very weak radio interference. Further adjustments were undertaken: on May, 5th the dead time after a detection of interference was increased to 5 minutes. On July, 10th the $\mathrm{C}++$ control software of the signal processing platform was updated with the second version of the RFI detection algorithm. Due to more stable performance of the interference detection and the different handling of the L1 and L5 frequency bands the dead time was reduced back to 30 seconds for the detected events at L1 frequency and kept at 5 minute for L5 signals.

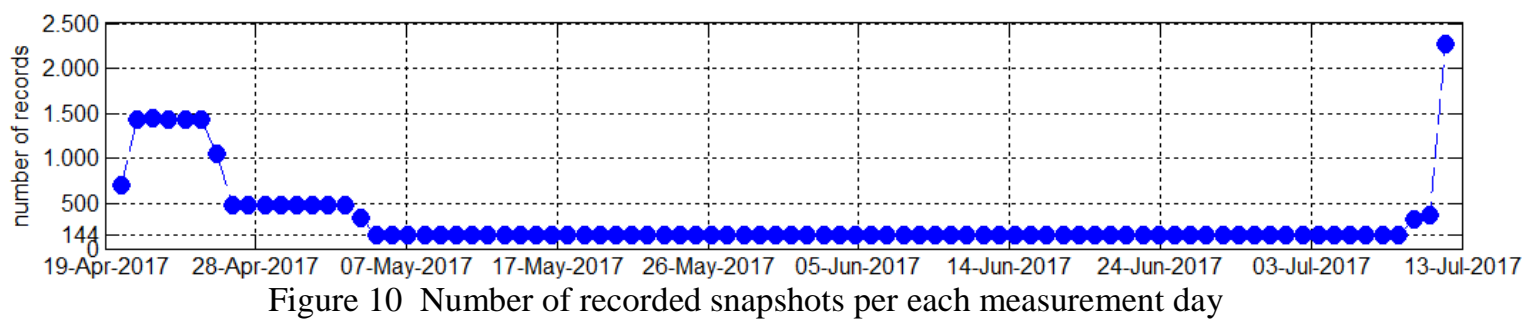

Each number of the recorded snapshots presented in Figure 10 consists of the records triggered by the RFI detection algorithm or by the periodical data control trigger. Figure 11 shows the portion of the records with detected man-made emissions in L1 or L5 bands with respect to the entire number of the records in a given day. It can be observed that the relative frequency of the record with detected interference significantly variates over time reaching 40\% in L1 band and 100\% in L5 band. The inspection of the logged PVT status information shows that the ship was mostly docked at a port during the time with higher occurrence of the interference in L1 band. It was close to the land when experienced the interference in L5 band.

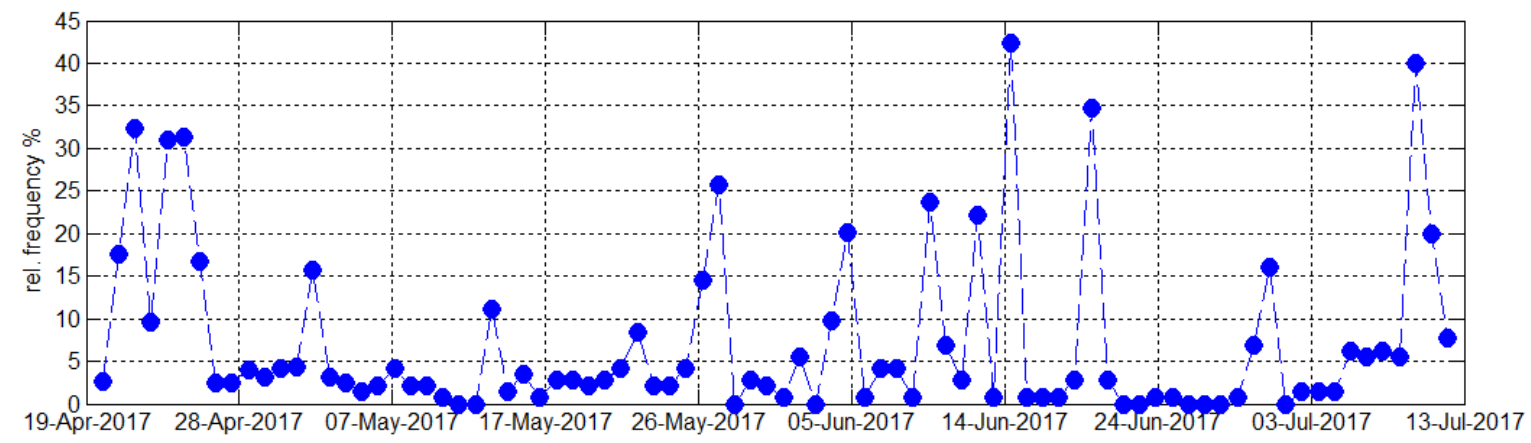

a) L1 band

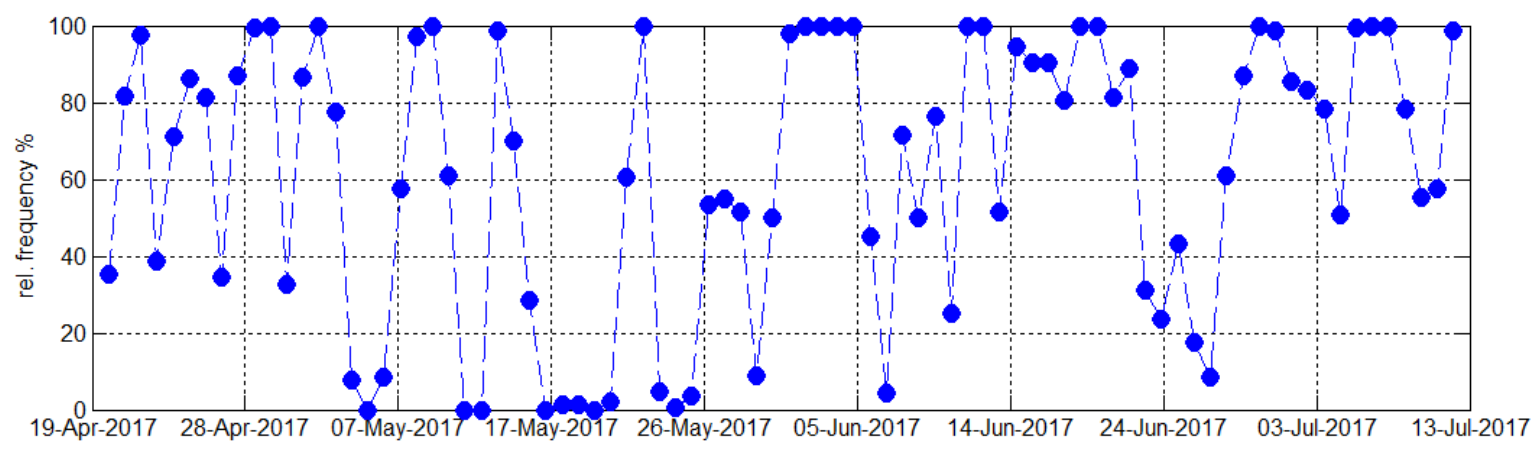

b) L5 band

Figure 11 Relative frequency of records with detected presence of man-made radio emissions

The entire numbers of the signal snapshots with the indication of the man-made emissions in the L1 and L5 GPS frequency bands are 3018 and 17602, correspondingly. For assessing the interference effect on the operation of a GNSS receiver the variation of the receiver power on the corresponding frequency band has been utilized. In order to make this assessment 
representative for a regular GNSS receiver using a single antenna, the variation of the received power has been observed in the signal path of the array element 7 that is the element on the top of the conformal antenna array (see Figure 4). The antenna gain provided by this array element at low elevations is comparable to the one of a typical commercial GNSS antenna. For the assessment, it is assumed that the increase of the received power occurs because of the presence of interference signals. Therefore, the increase of the received power results in the corresponding raise of the effective noise level and drop of the effective carrier-to-noise density ratio (C/N0). The time evolution of the received power at the output of array element number 7 in L1 band during the measurement campaign is shown in Figure 12. Three stages of the assessed interference effect - weak, medium and strong - have been set by using the estimated drop of effective C/N0 as the metric. The corresponding discrimination levels for the drop of $\mathrm{C} / \mathrm{N} 0$ are given in Table 1 along with the number of the RFI events belonging to the corresponding categories.

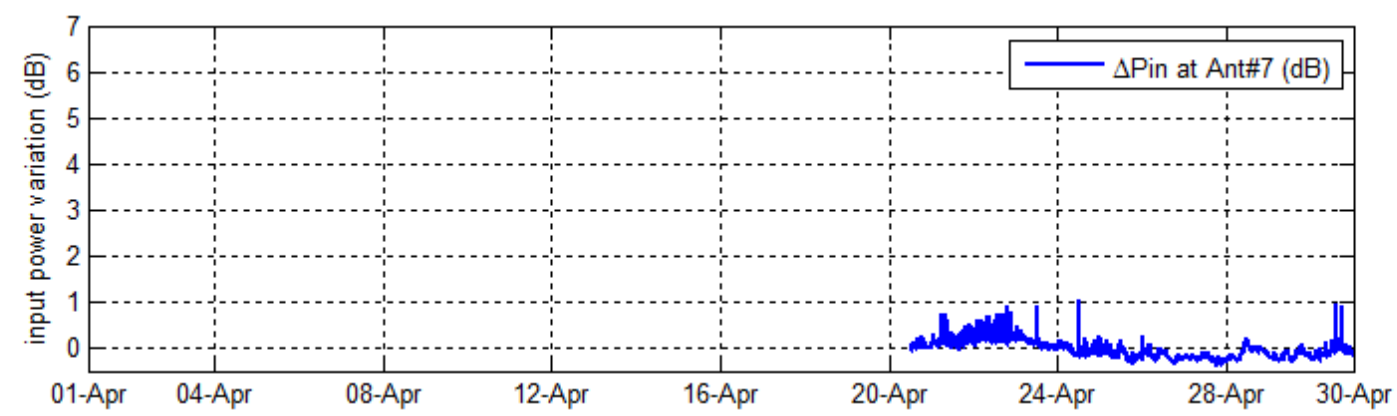

a) from start of measurements on April, 20th until the end of April of 2017

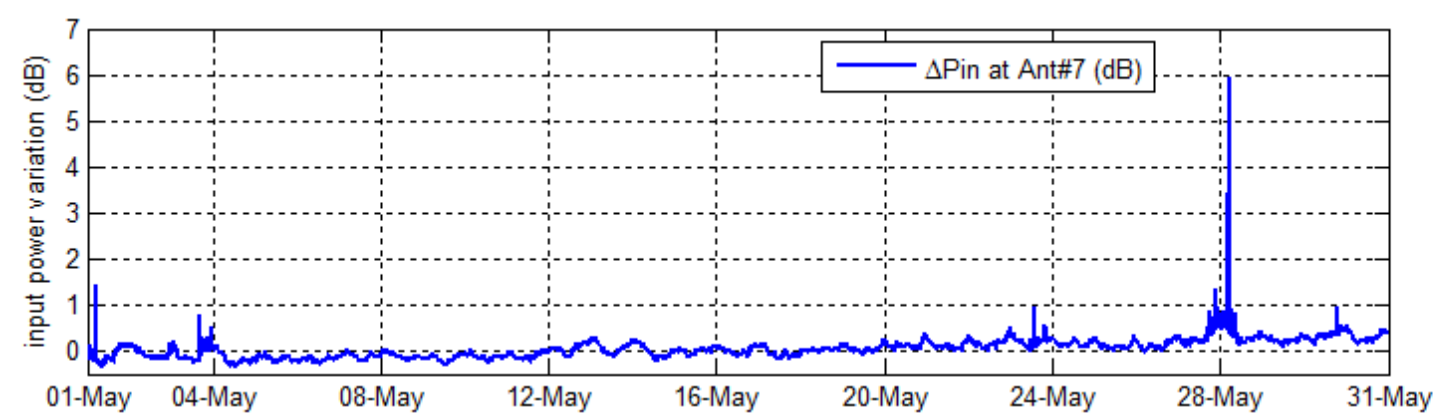

b) throughout May of 2017

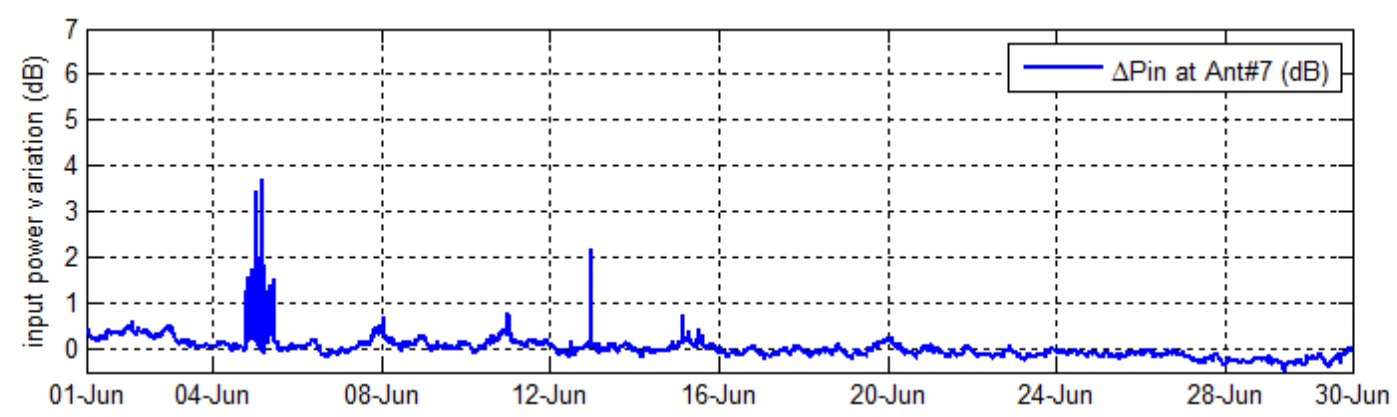

c) throughout June of 2017

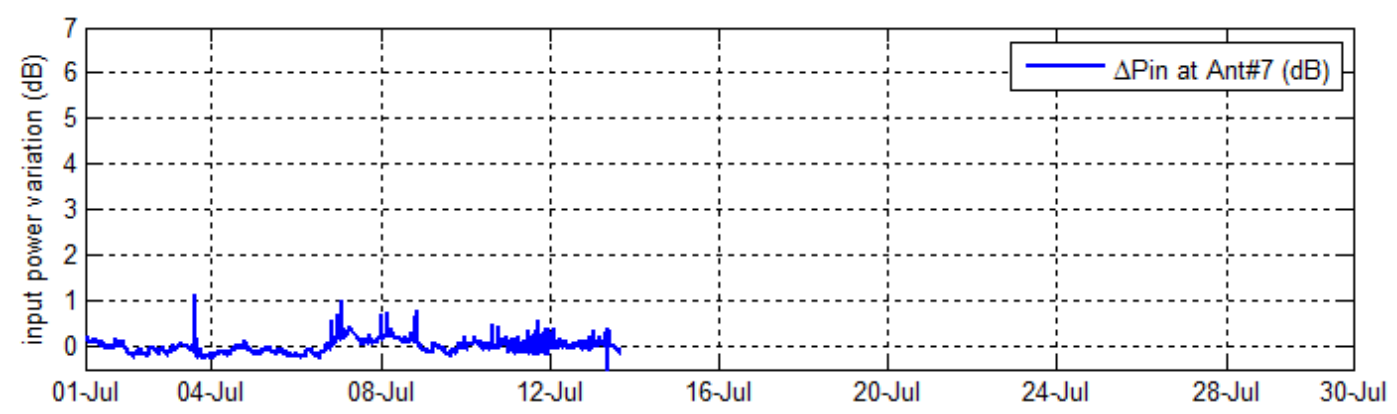

d) from beginning of July until the end of the first route of the ship on July, 13rd 2017

Figure 12 Variation of received power in L1 band 
It can be observed from Table 1 that the actual number of the collected IF signal snapshots indicating a potentially significant RFI effect in GPS L1 band is quite low. Only in a single snapshot recorded on May, 28th the rise of the received IF power is above level of $5 \mathrm{~dB}$. All other detected occurrences of the potential radio frequency interference fall into the categories medium (3 incidents) and weak (21 incidents) according to the proposed classification. Please note that the corresponding signals records were recorded in a few compact time windows. The analysis of those records shows that the same type of a man-made emission was observed within a single time window. Some of those emissions were observed for several hours, with 15 hours being the longest observation time on June, 4th-5th. Other emissions could be observed only in several signal records like on June, 12th, where the interference signal was present during 40 minutes. Over the entire timespan of the measurement campaign four time windows with the presence of radio frequency interference can be identified:

(i) May, 28th, duration of 1 hour;

(ii) June, 4th-5th (GPS time), duration of 15 hours;

(iii) June, 12th, duration of 40 minutes;

(iv) July, 3rd, duration of 1 hour 50 minutes.

More details on these RFI events will be presented in the following (see Figure 14 - Figure 17).

Table 1 Number of signal records indicating a potentially significant interference effect

\begin{tabular}{llrr}
\hline \multicolumn{1}{c}{ Interference effect } & L1 band & L5 band \\
\hline weak, & $\Delta C / N_{0} \in[-1,-3[\mathrm{~dB}$ & 21 & 2101 \\
medium, $\Delta C / N_{0} \in[-3,-5[\mathrm{~dB}$ & 3 & 3602 \\
strong, $\Delta C / N_{0} \leq-5 \mathrm{~dB}$ & 1 & 4317 \\
\hline
\end{tabular}

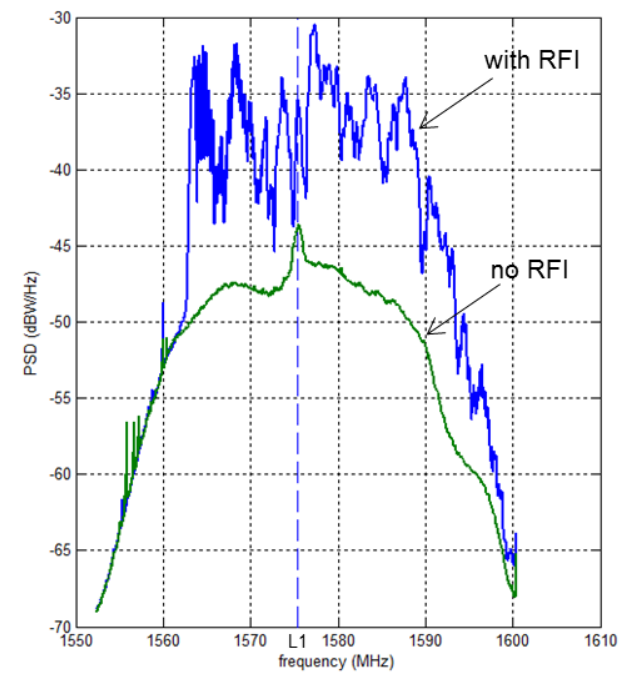

a) power spectrum density

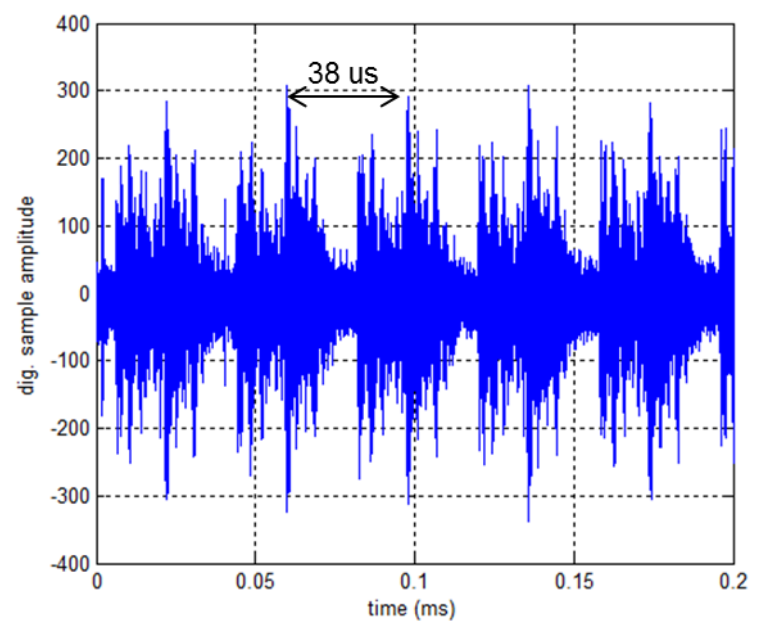

c) time plot

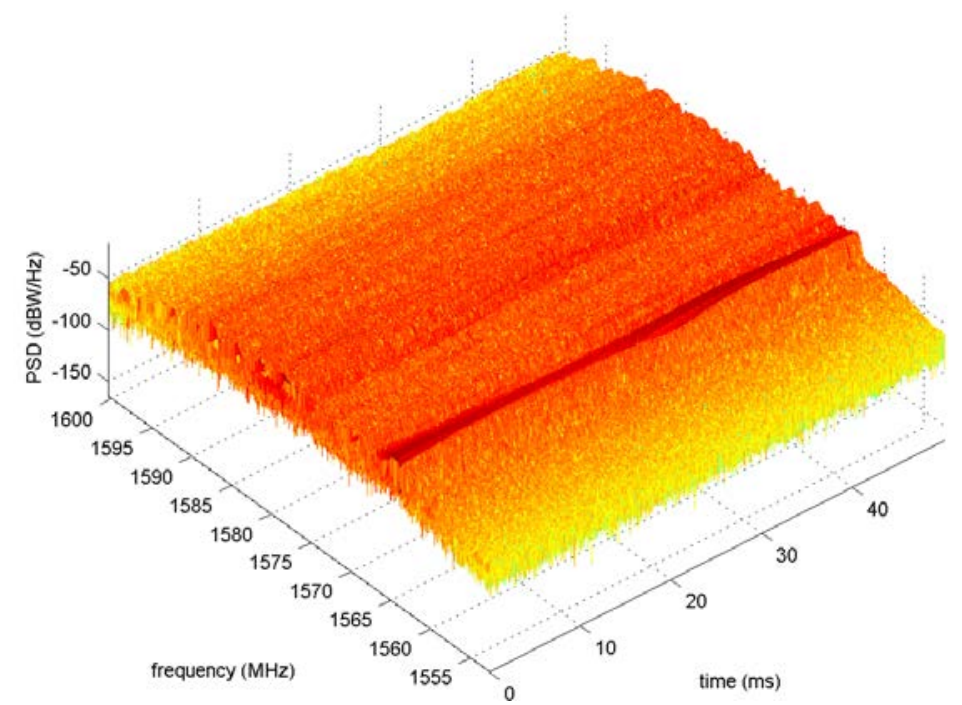

b) spectrogram

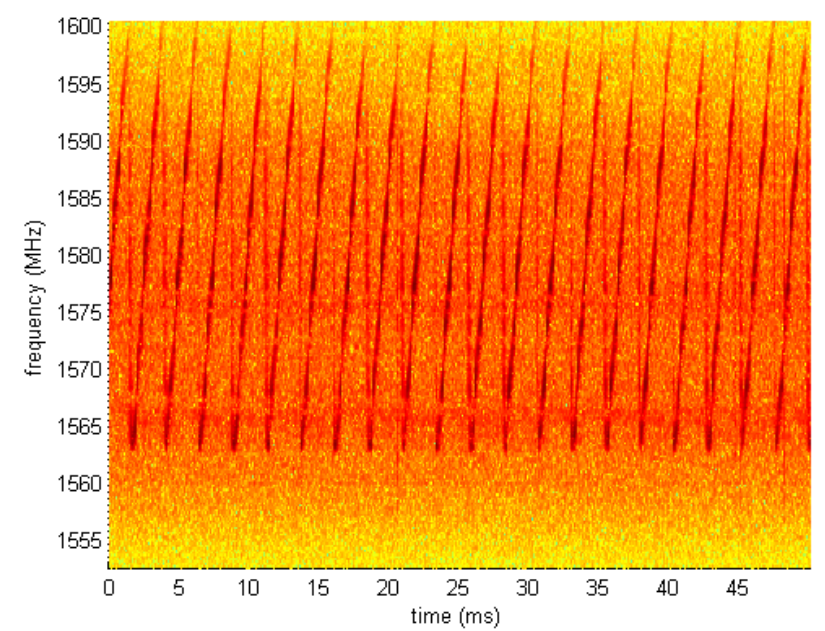

d) spectrogram, top view to show frequency sweep

Figure 13 Characteristics of RFI signal at L1 band detected on May, 28 
The plots with the power spectrum density (PSD), spectrograms (i.e. a time evolution of PSD) and the time evolution of the signal amplitude for the interference signal that was detected on May, 28th are presented in Figure 13 . These plots were obtained by using the signal record with the highest observed power of the RFI signal at 5:15 UTC (see Figure 14a). For the comparison reasons, in the PSD plot (see Figure 13a) a second curve corresponding to interference-free case is also plotted. A clear increase of the power spectrum density of up to approximately $10 \mathrm{~dB}$ over the large part of the L1 band spectrum can be observed. The spectrogram in Figure 13b does not indicate a distinctive pulsed character of the interference. However when inspecting the time plot of the signal in Figure 13c, a periodical structure of the signal with some pulsed behavior can be observed. The measured period of the signal is $38 \mu \mathrm{s}$. The top view of the spectrogram (see Figure 13d) clearly shows that the instant frequency of the signal is sweeping between $1563 \mathrm{MHz}$ and $1600 \mathrm{MHz}$. It can be observed that the fast sweep of the instant frequency does not allow to completely resolve the periodical structure of this chirp signal (please compare the signal repetition time in Figure 13c and the sweep period in Figure 13d). Also, the sweep time cannot be correctly determined by using only the spectrogram.

The interference effect of the RFI signal observed on May, 28th is illustrated in Figure 14 by utilizing two figures of merit: the mean C/N0 value averaged over all tracked GPS L1 satellites and the number of tracked satellites.

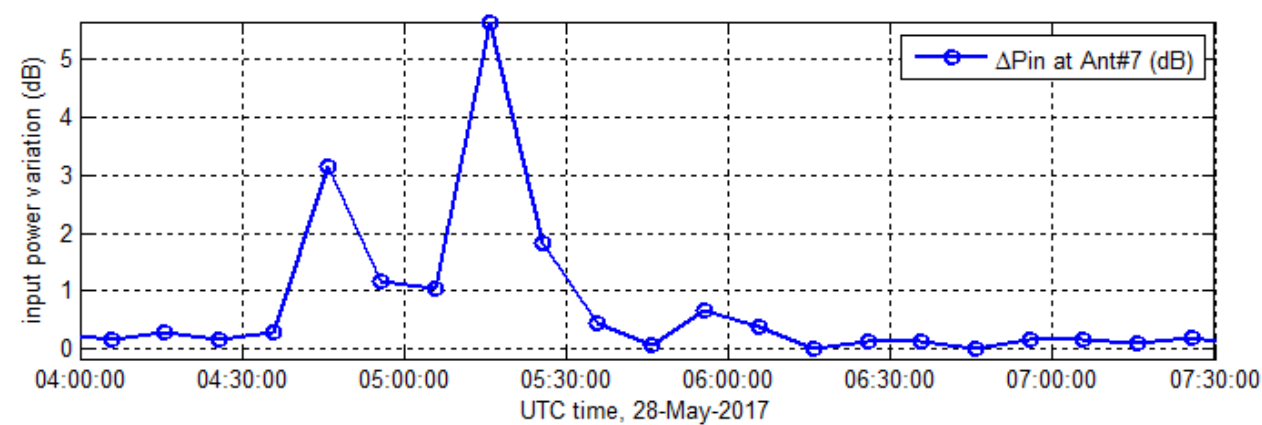

a) variation of received power

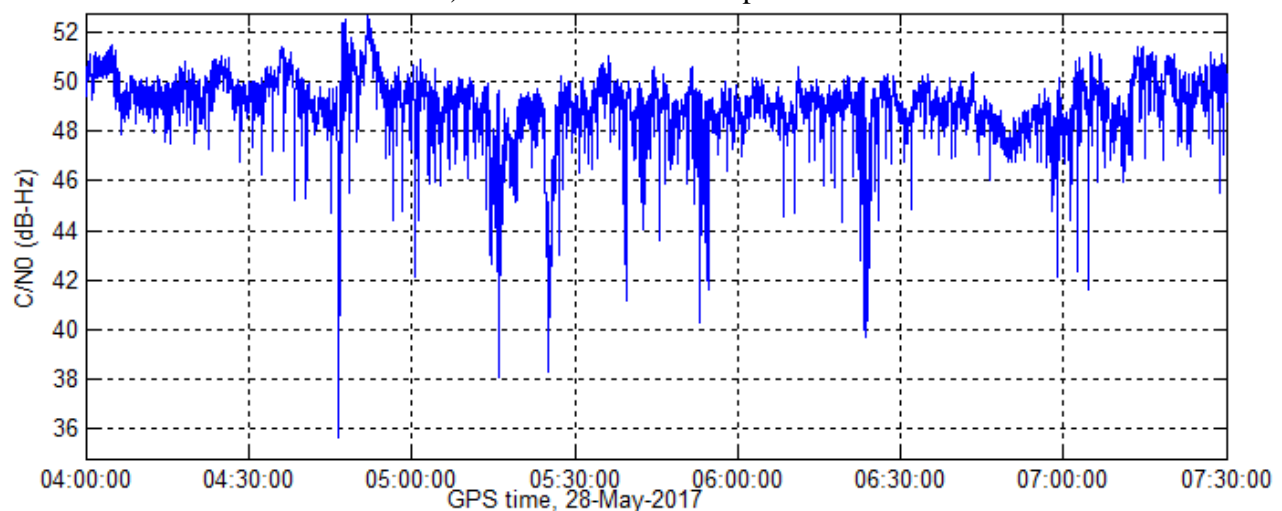

b) mean C/N0 averaged over all satellites in track

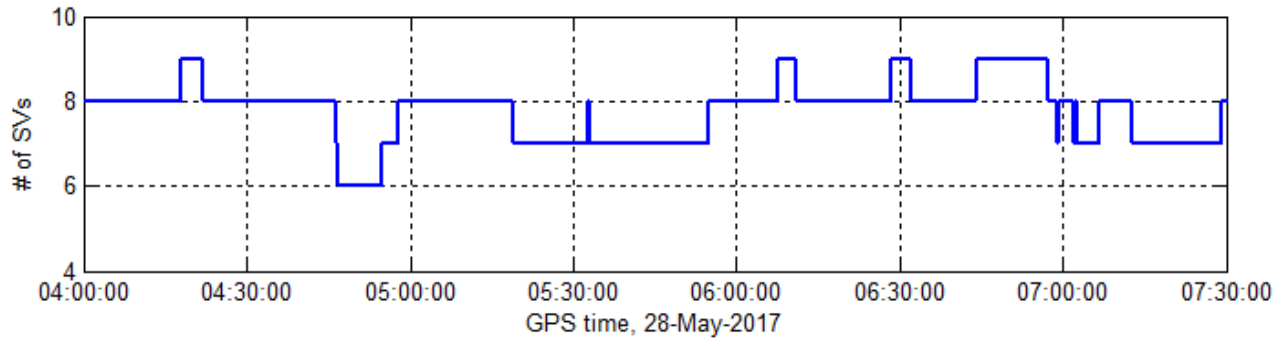

c) number of GPS L1 satellites in track

Figure 14 Effect of strongest detected RFI at L1 band

on mean $\mathrm{C} / \mathrm{N} 0$ and number of tracked satellites observed by the real-time multi-antenna receiver

By inspecting the plots in Figure 14a Figure 14b it can be concluded that the drops of the mean C/N0 at 4:15 UTC and at 5:15 UTC are very likely triggered by the radio interference. Also the loss of lock for two satellite signals at 4:15 UTC can be connected to the RFI event. Other drops of the mean C/N0 can also be triggered by the interference. This cannot be clearly 
identified since the corresponding moments fall into the dead time between two signal records. Another possible reason for the changes of the mean C/N0 may be the blockage of the GPS satellites signals by the container crane passing over the conformal antenna array. According the position status log, the container ship was docked in a sea port in the considered time and no PVT solutions are missing. Considering the short duration of the $\mathrm{C} / \mathrm{N} 0$ degradations it can be concluded that the cumulative interference effect was quite moderate. Therefore it was not resulted in the loss of lock of larger number of satellites and the outage of the positioning and timing information delivered by the receiver.

The signal characteristics of other detected interference events are presented in Figure 15- Figure 17. Because the powers of these interference signals are significantly lower than in the case of the chirp-type interference discussed above, no noticeable effect on the real-time receiver is observed. Therefore no figures of merit for the real-time receiver are presented in Figure 15Figure 17.

The interference detected on June, 4th is a of continuous narrow-band signal type (see Figure 15b). The carrier frequency of the signal jumps after some time from 1580.2 MHz to 1569.7 MHz as shown in Figure 15a.

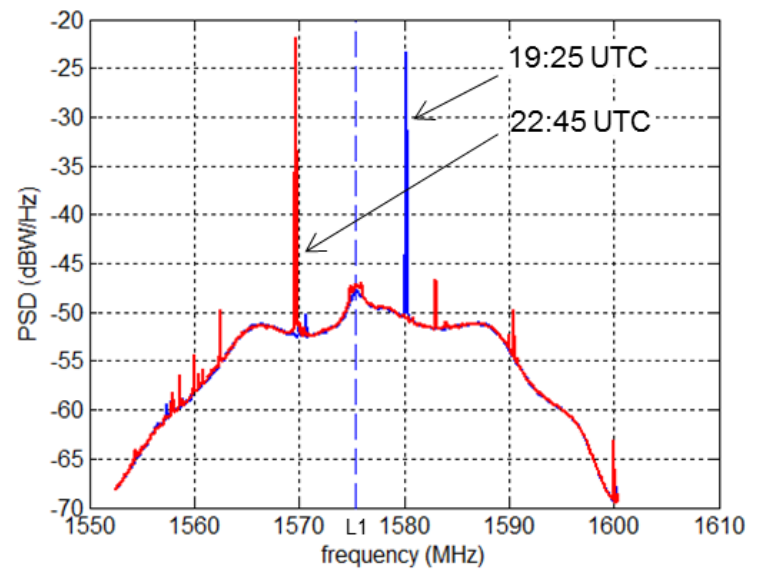

a) power spectrum density

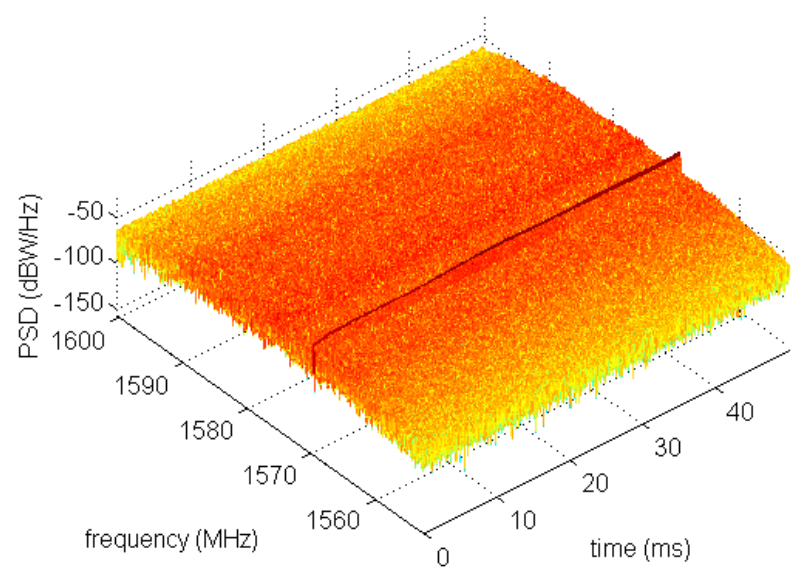

b) spectrogram, 22:45 UTC

Figure 15 Characteristics of RFI signal at L1 band detected on June, 4

A continuous partial-band interference that was detected on June, 12 is shown in Figure 16. The signal has bandwidth of 1.6 MHz which is centered on $1585 \mathrm{MHz}$.

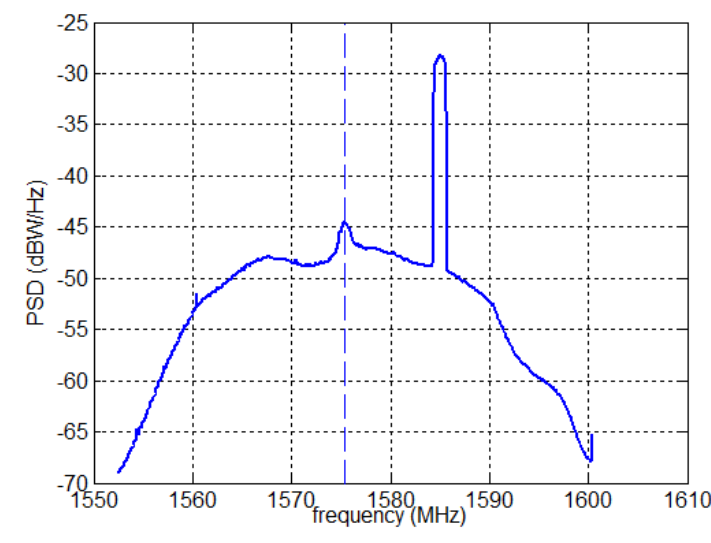

a) power spectrum density

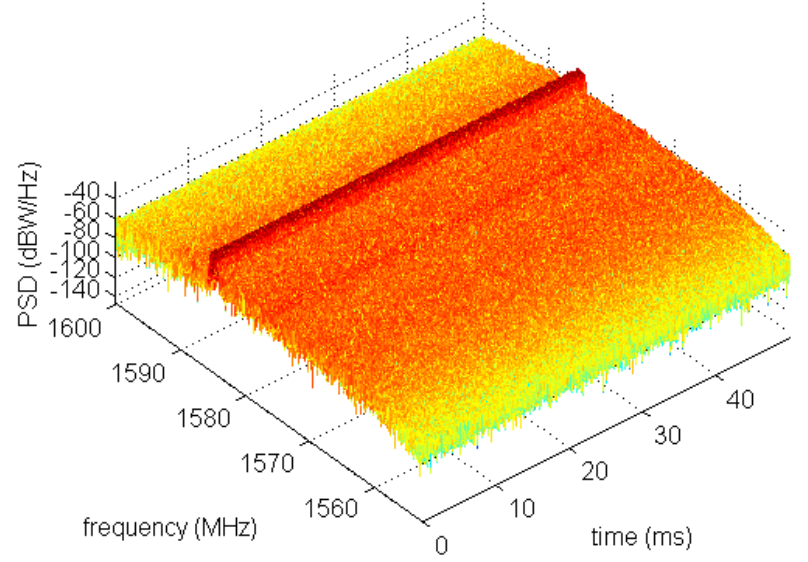

b) spectrogram

Figure 16 Characteristics of RFI signal at L1 band detected on June, 12

A wise-band continuous chirp-type interference detected on July, 3 is shown in Figure 17. The sweep time of the signal is $12 \mu$ s (see Figure 17b). Because of the fast sweep time and relatively low power of the signal, its periodic structure is not observable by using spectrograms. 


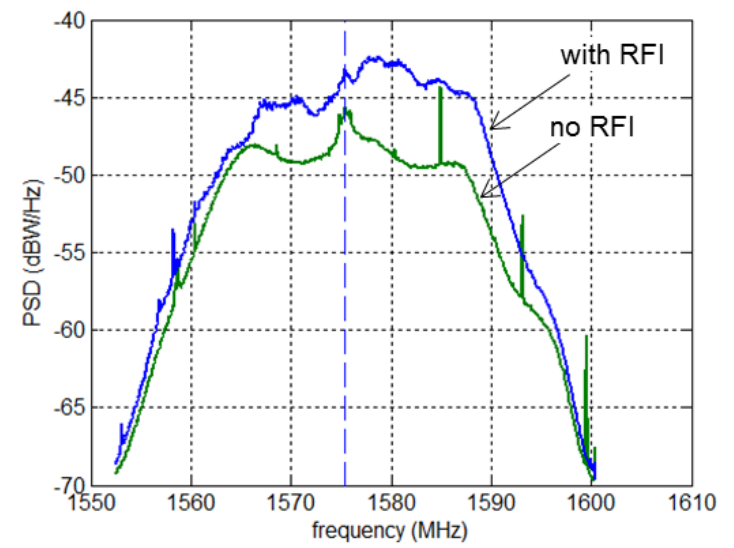

a) power spectrum density

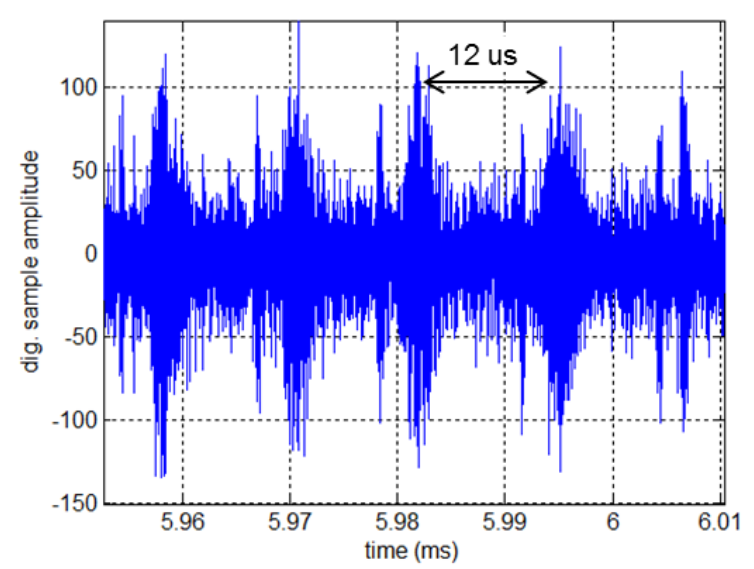

b) time plot

Figure 17 Characteristics of RFI signal at L1 band detected on July, 3

Until now the discussion about the detected interference events was focused on the situation in GPS/Galileo L1/E1 band. In the rest of this chapter a short overview of the detected man-made emissions in GPS/Galileo L5/E5a band will be given. First, the signal situation in L5 band during the strong L1 interference event on May, 28th will be examined. The corresponding plots of the signal characteristics are presented in Figure 18.

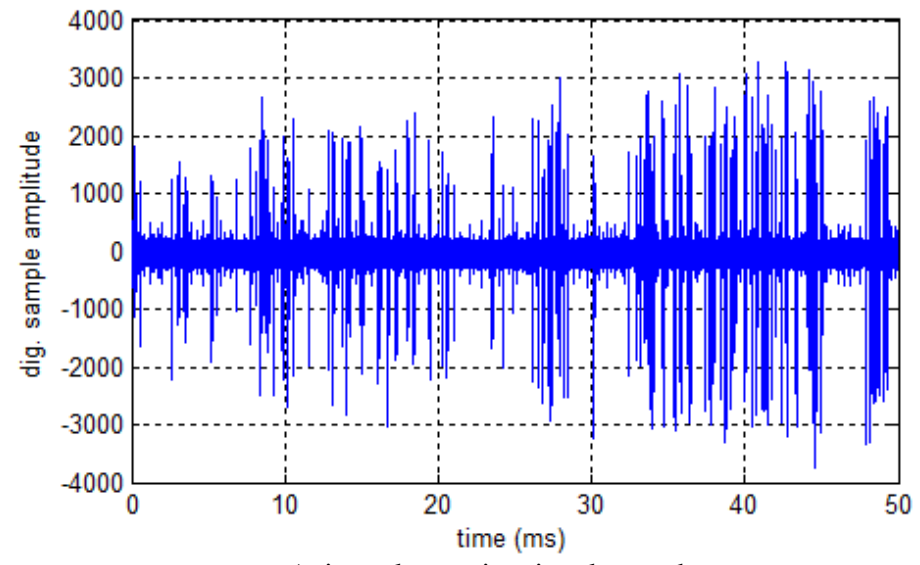

a) time plot, entire signal record

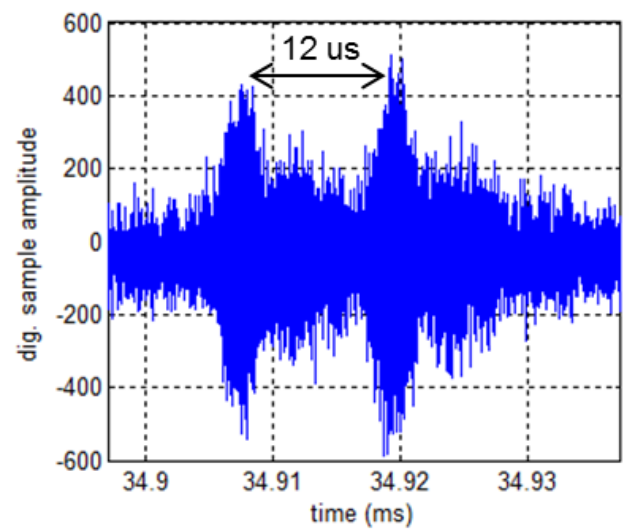

c) time plot, zoomed

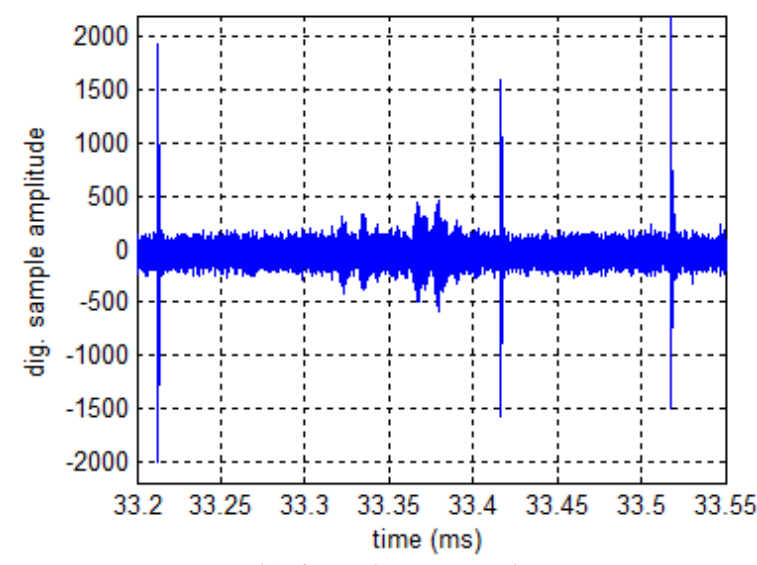

b) time plot, zoomed

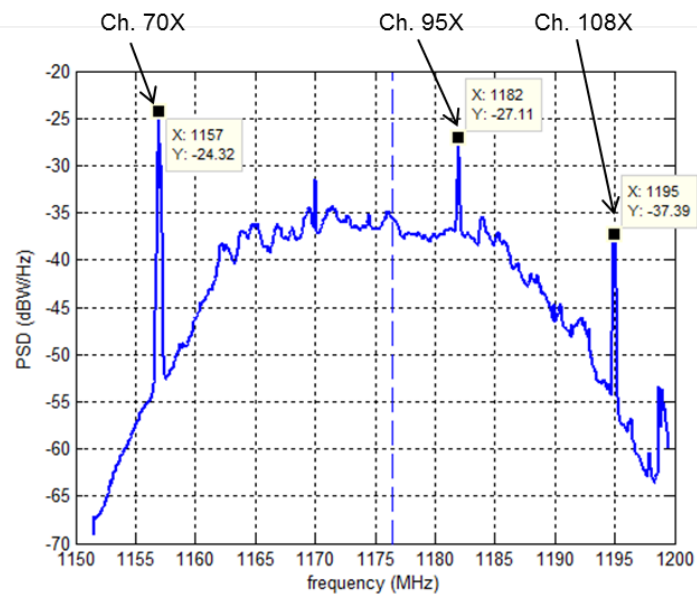

d) power spectrum density

Figure 18 Signal situation at L5 band, May, 28th 
It can be observed that the time plot of the signal is populated by a large number of pulses (see Figure 18a). A zoomed view (see Figure 18b) shows that these pulses are of two types - very short chirp pulses with the duration of $1.2 \mu$ s and several pulse pairs. The latter can be identified as the signal of the distance measurement equipment (DME), a radio navigation system used in aviation. A zoomed view of one of the DME pulse pairs is shown in Figure 18c. The measured separation of two pulses in a pair - $12 \mu \mathrm{s}$ - matches exactly the corresponding figure given by the DME signal specifications for the X-mode [13]. The spikes in the power spectrum observed in Figure 18d clearly match the carrier frequencies of the DME channels which are used by the DME ground stations (transponders). The corresponding DME ground stations operating on the identified channels can be actually found in the neighborhood of the container ship in the considered time (see Figure 19).

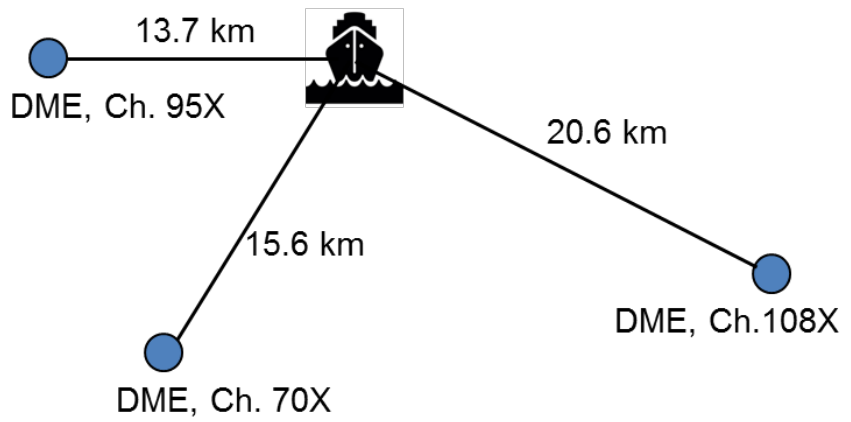

Figure 19 Relative locations of the closest DME stations on May, 28th

The signal characteristics presented in Figure 18 indicate that the presence of the signals of other radio systems operating in L5 band may cause significant interference to the GNSS navigation signals. Similar to the discussion regarding L1 band, the potential interference effect can be assessed by examining the increase of the received IF power. The plots for the variation of the received power in L5 band during May and June 2017 are shown in Figure 20. In these plots it can be observed that the power level of the received signals can rise up to more than $20 \mathrm{~dB}$, which is much larger than the corresponding figure for the strongest detected interference in L1 band. In order to get some more details about the signal situation to be encountered by a GNSS L5/E5a receiver, the signal records from May 2017 delivering the highest received power measurement have been examined. The corresponding results are presented in Figure 21, Figure 22 and Figure 23.

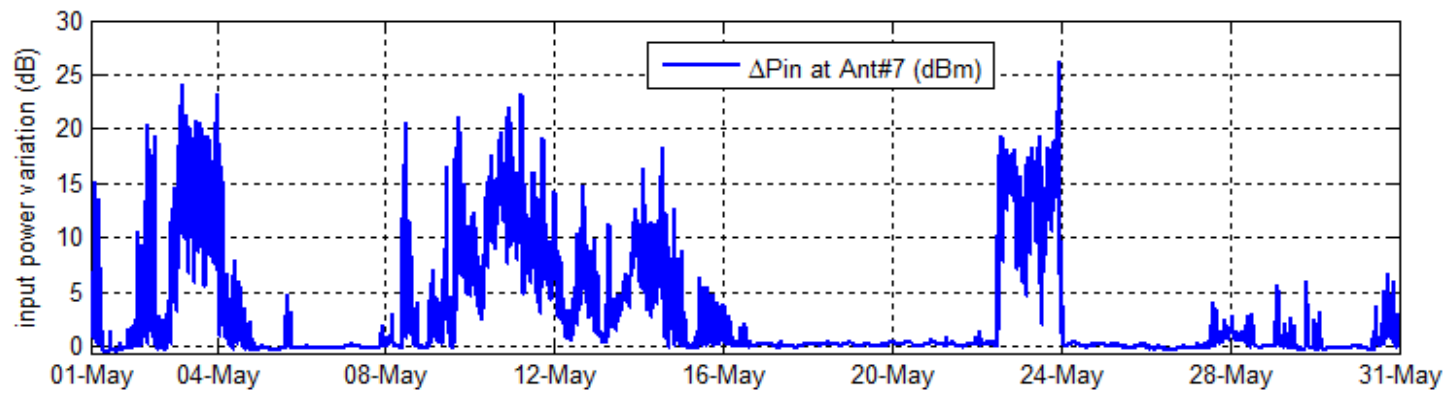

a) throughout May of 2017

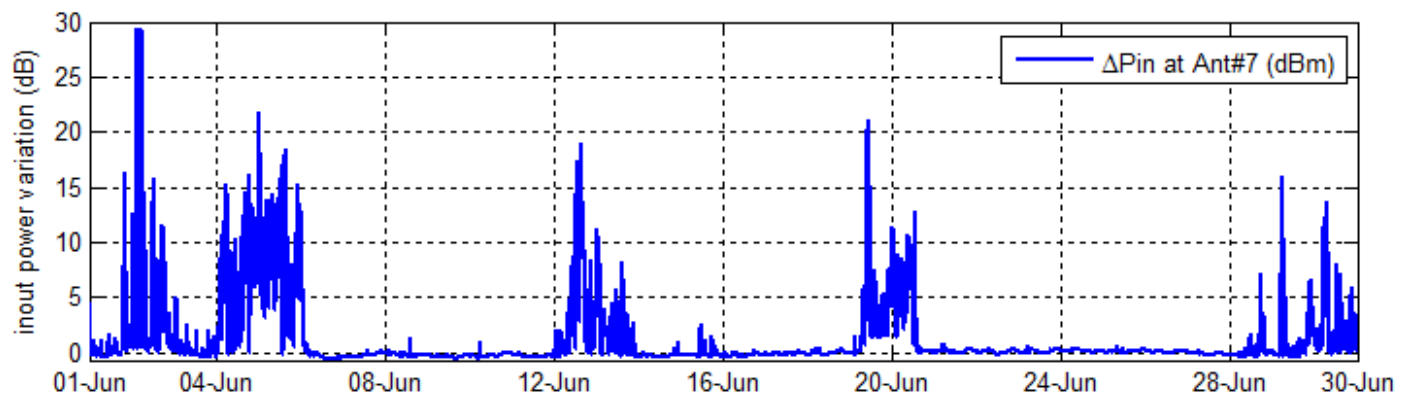

b) throughout June of 2017

Figure 20 Variation of received power in L5 band 
The signal characteristics plotted in Figure 21, Figure 22 and Figure 23 indicate the presence of the signals of DME ground stations in all three selected days of May 2017. Though the DME pulses are not the only type of the man-made emissions in L5 band (see Figure 18), their contribution to the received IF power in the examined cases turns out to be dominant. This interference type is well known to the GNSS community (see for example [7]) and several appropriate mitigation techniques have been investigated.

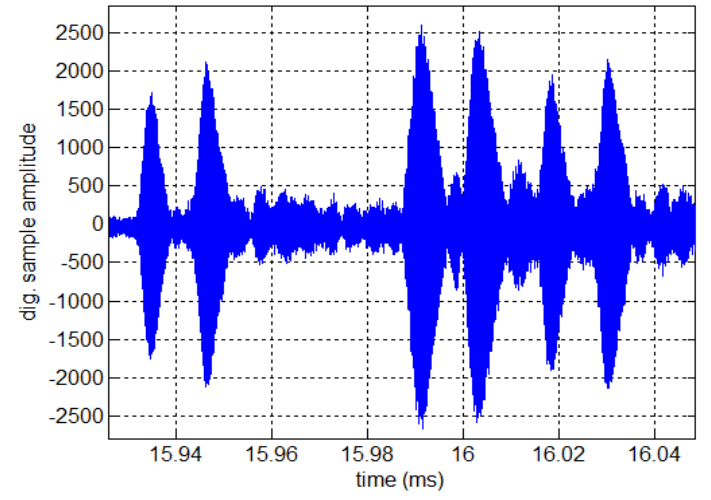

a) time plot

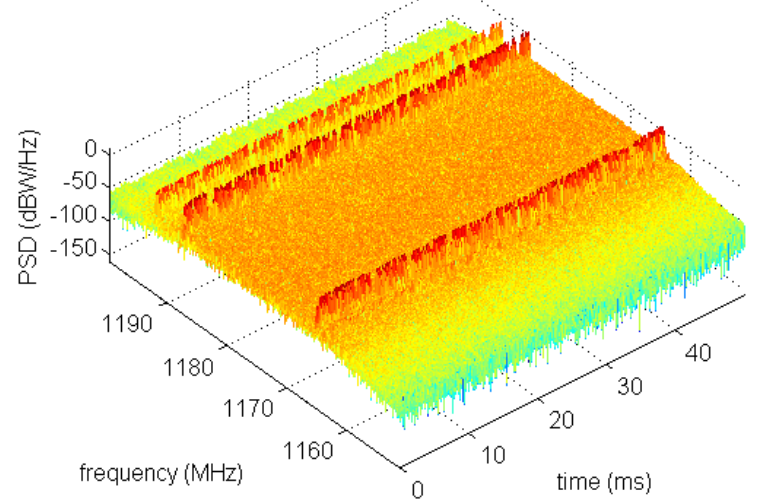

b) spectrogram

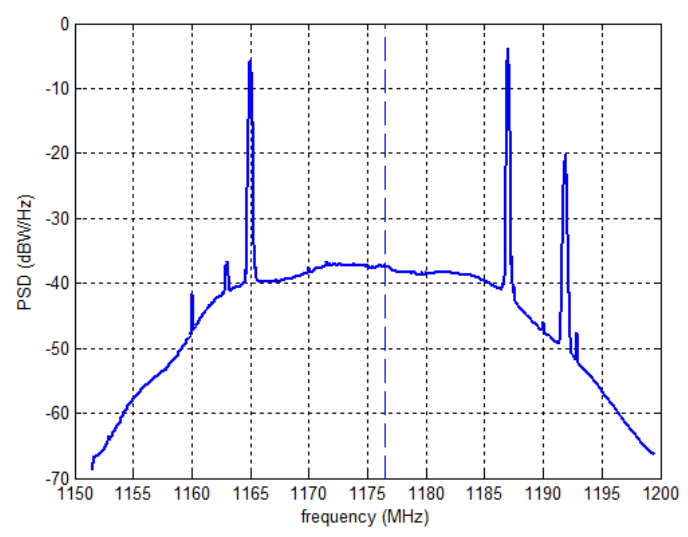

c) power spectrum density

Figure 21 Signals at L5 band, 3-May,

received power increased by $20 \mathrm{~dB}$, estimated duty cycle of $9.7 \%$

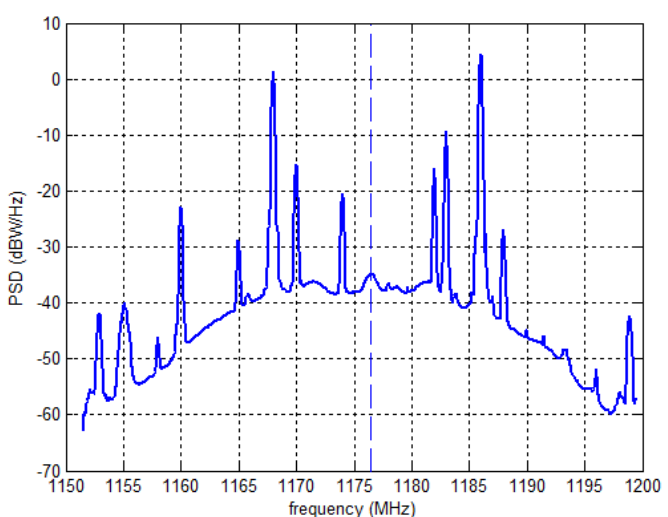

a) power spectrum density

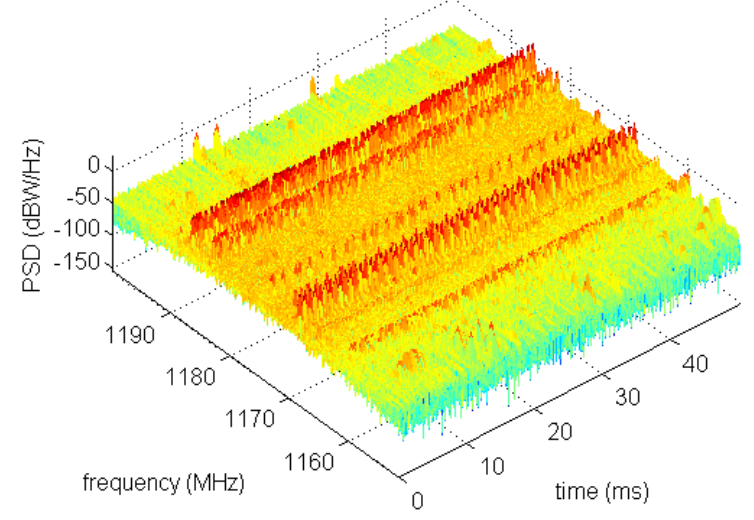

b) spectrogram

Figure 22 Signals at L5 band, May, 10, received power increased by $23 \mathrm{~dB}$, estimated duty cycle of $11.9 \%$

One of the most practical countermeasures against the interference introduced by the DME signals is to use a digital pulse blanking technique [15]. In order to assess the portion of the GNSS signal that is going lost after applying pulse blanking, the 
duty cycles of the pulse blanker has been estimated. The highest duty cycle of $11.9 \%$ has been obtained for the signal record from on May, 10th (see Figure 22). Even accounting for the fact that not all energy of the pulsed interference can be effectively removed by the pulse blanking technique, the obtained duty cycles values suggest that the resulting degradation of the C/N0 in the tracking loops of the GNSS L5/E5a receiver will be still tolerable.

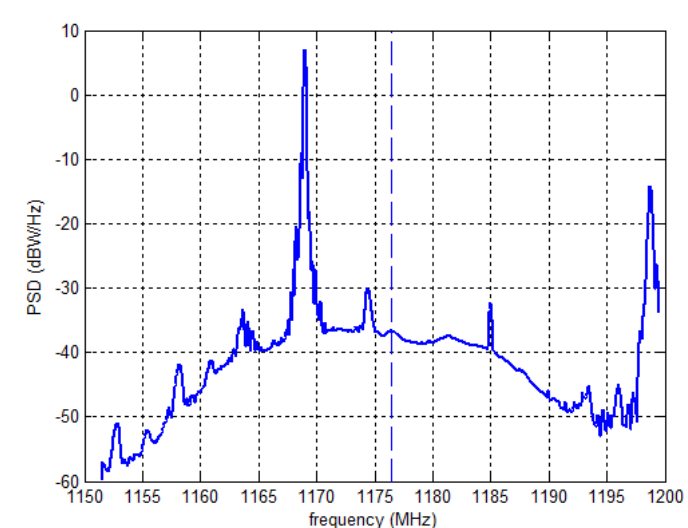

a) power spectrum density

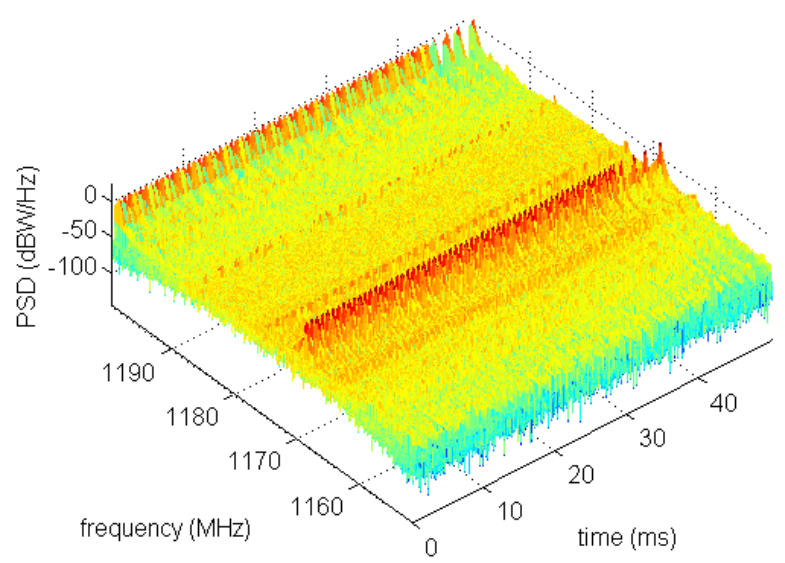

b) spectrogram

Figure 23 Signals at L5 band, May, 23rd, received power increased by $26 \mathrm{~dB}$, estimated duty cycle of $8.9 \%$

\section{CONCLUSIONS}

In this paper the results of the maritime interference measurement campaign carried out during 2.5 months on board of a large container ship serving the route between large seaports in the Mediterranean, Arabian Peninsula and East Asia have been presented. The collected data allows taking a deeper look at the interference situation in GPS/Galileo L1/E1 and L5/E5a frequency bands where the open service navigation signals of these two GNSSs are allocated. At the moment of writing the paper, the developed measurement system is still in operation on the board of the ship. The additional data collected in the next measurement runs will be used to obtain a more representative picture of the maritime signal environment.

The first results of the analysis of the collected data show that radio frequency interference is a part of the signal environment encountered by a GNSS receiver in the harbors or in the vicinity of the land. However the assessed interference effect on the navigation signals in L1 band is turned out to be quite small. Only in rare cases the estimated degradation of C/N0 exceeds $1 \mathrm{~dB}$ level. Four such interference events have been found so far, while only for only one of them the estimated C/N0 degradation is above $5 \mathrm{~dB}$ level. All the detected RFI events in L1 band occurred when the ship was docked in a harbor. No outage of the positioning service was observed in this time either by the multi-antenna receiver integrated in the measurement system or by the receivers used by project partners.

The signal situation in L5 band is determined to the large degree by the strong pulsed signals of DME system. These signals can cause strong interference effect. The observed increase of the received IF power in L5 band can exceed $20 \mathrm{~dB}$. The estimated duty cycle of a digital pulse blanker applied for mitigation of this pulsed interference can achieve values slightly above $10 \%$. Such duty cycles should result only in moderate degradations of C/N0. However, the receiver signal processing chain, in the first turn the ADCs and the active components of the RF front end, should be designed to tolerate such high signal dynamic and deliver short relaxation times after arriving of strong pulsed signals.

The processing of the available data is still ongoing. The next work will focus on the detailed analysis and classification of the different types of man-made emissions identified in the collected data. Also the signal processing in the spatial domain will be used to fully explore the information provided by the multiple signals of the conformal antenna array.

\section{ACKNOWLEDGMENTS}

The research which results are reported in this paper was performed in the frame of siNafa project funded by the German Federal Ministry for Economic Affairs and Energy through the project executing organization Jüllich. This support is greatly acknowledged. The authors also gratefully acknowledge the support of Hapag Lloyd Company that made the measurement campaign possible. We would also like to thank the project partners from navXperience, Raytheon Anschütz and Technical University of Berlin for the fruitful cooperation. 


\section{REFERENCES}

[1] A. Parkins, A. Grant, and P. Cross, "The impact of new signals on precise marine navigation - initial results from an experiment in Harwich harbour.” pp. 1-14, 2008.

[2] A. Grant, P. Williams, N. Ward, and S. Basker, “GPS Jamming and the Impact on Maritime Navigation,” J. Navig., vol. 62, no. 2, pp. 173-187, 2009.

[3] IMO Sub-Committee on Safety of Navigation, "Development of an e-Navigation strategy implementation plan.” 2011.

[4] IALA, "IALA Recommendation R-129 on GNSS Vulnerability and mitigation measures.” pp. 1-19, 2008.

[5] “Assessment of Radio Frequency Interference Relevant to the GNSS (RTCA/DO-235A),” RTCA, Inc., 2002.

[6] J. R. Clynch, A. A. Parker, R. W. Adler, and W. R. Vincent, "System challenge-The hunt for RFI-Unjamming a Coast Harbor,” GPS World, pp. 16-22, 2003.

[7] FBI Cyber Division, “Cargo Thieves use GPS jammers to Mask GPS Trackers,” no. 2 October, pp. 1-4, 2014.

[8] M. Cuntz, A. Konovaltsev, M. Heckler, A. Hornbostel, L. Kurz, G. Kappen, and T. Noll, "Lessons Learnt: The Development of a Robust Multi-Antenna GNSS Receiver,” in Proc. ION GNSS 2010.

[9] A. Konovaltsev, M. Cuntz, L. A. Greda, M. V. T. Heckler, and M. Meurer, "Antenna and RF front end calibration in a GNSS array receiver, "in 2010 IEEE International Microwave Workshop Series on RF Front-ends for Software Defined and Cognitive Radio Solutions (IMWS), Feb 2010, pp. 1-4.

[10] M. V. T. Heckler, M. Cuntz, A. Konovaltsev, L. A. Greda, A. Dreher, and M. Meurer, "Development of Robust Safety-ofLife Navigation Receivers,” IEEE Trans. Microw. Theory Tech., vol. 59, no. 4, pp. 998-1005, Apr. 2011.

[11] P. Ward, J. Betz, C. Hegarty, “Chapter 5: Satellite Signal Acquisition, Tracking, and Data Demodulation” in Understanding GPS: principles and applications, 2nd Edition, E. D. Kaplan and C. Hegarty, Eds. Norwood, MA: Artech House, 2005, p. 726.

[12] G. Dillard, “A Moving-Window Detector for Binary Integration”, IEEE Tranc. on Information Theory, Vol. IT-13, No. 1, Jan. 1967, pp. 2-6.

[13] U.S. Department of Commerce, National Telecommunications and Information Administration, Manual of Regulations and Procedures for Federal Radio Frequency Management, Chapter 4: “Table of Frequency Allocation Footnotes \& Allotments”, Edition May 2013, 806 p.

[14] G. Gao, "DME/TACAN Interference and its Mitigation in L5/E5 Bands," in Proc. of ION GNSS 2007, Fort Worth, TX, September 2007, pp. 1191-1200.

[15] J. Grabowski and C. Hegarty, “Characterization of L5 receiver performance using digital pulse blanking,” in Proc. of ION GPS, Portland, OR, Sept. 2002, pp. 1630-1635. 